\title{
Time-resolved infrared absorption spectroscopy applied to photoinduced reactions: how and why
}

\author{
Alberto Mezzetti ${ }^{1}\left[\right.$ ] Josefine Schnee ${ }^{1} \cdot$ Andrea Lapini $^{2,3} \cdot$ Mariangela Di Donato ${ }^{2,4}$
}

Received: 30 October 2021 / Accepted: 28 January 2022 / Published online: 21 February 2022

( ) The Author(s), under exclusive licence to European Photochemistry Association, European Society for Photobiology 2022, corrected publication 2022

\begin{abstract}
Time-resolved infrared (IR) spectroscopy is a widely used technique in the investigation of photoinduced reactions, given its capabilities of providing structural information about the presence of intermediates and the reaction mechanism. Despite the fact that it is used in several fields since the ' $80 \mathrm{~s}$, the communication between the different scientific communities (photochemists, photobiologists, etc.) has been to date quite limited. In some cases, this lack of communication happened-and still happens-even inside the same scientific community (for instance between specialists in ultrafast ps/fs IR and those in "fast" ns/ $\mu \mathrm{s} / \mathrm{ms}$ IR). Even more surprising is the difficulty of non-specialists to understand the potential of time-resolved IR spectroscopy, despite the fact that IR spectroscopy is normally taught to all chemistry and material science students, and to several biology and physics students. This tutorial review aims at helping to solve these issues, first by providing a comprehensive but reader-friendly overview of the different techniques, and second, by focusing on five "case studies" (from photobiology, gas-phase photocatalysis, photochemistry, semiconductors and metal-carbonyl complexes). We are confident that this approach can help the reader-whichever is its background-to understand the capabilities of time-resolved IR spectroscopy to study the mechanism of photoinduced reactions.
\end{abstract}

\section{Graphical Abstract}

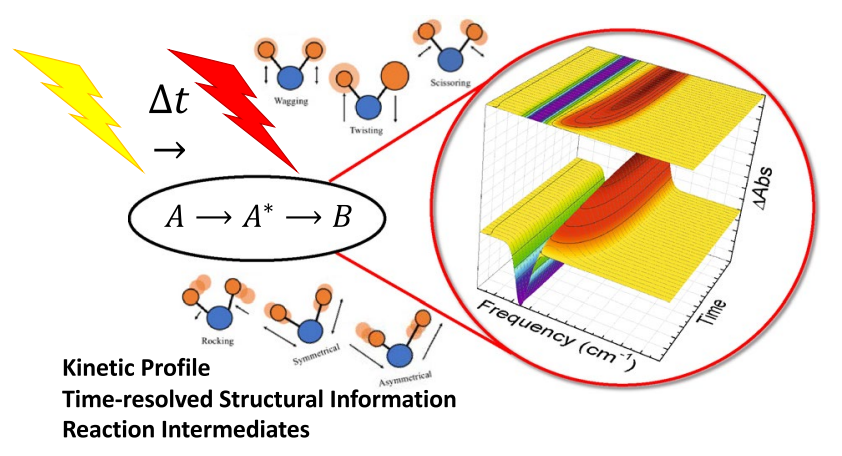

Alberto Mezzetti

alberto.mezzetti@sorbonne-universite.fr

Mariangela Di Donato

didonato@lens.unifi.it

1 Laboratoire de Réactivité de Surface (LRS), UMR 7197, Sorbonne Université, Campus Pierre et Marie Curie, 4 Pl Jussieu, 75005 Paris, France
2 European Laboratory for Non Linear Spectroscopy (LENS), via N. Carrara 1, 50019 Sesto Fiorentino, Italy

3 Dipartimento di Scienze Chimiche della Vita e della Sostenibilità Ambientale, Parco Area delle Scienze 17/A, 43124 Parma, Italy

4 ICCOM-CNR via Madonna del Piano 10, 50019 Sesto Fiorentino, Italy 


\section{Introduction}

Infrared (IR) absorption spectroscopy is a well-established technique to investigate physical, chemical and biochemical processes at an atomic scale. It is particularly informative when used in a time-resolved approach, i.e., following the evolution of the system under study with time. Compared to other spectroscopic techniques, IR spectroscopy offers the advantage of providing spectral contributions from all (or almost all) the constituents of the system (catalyst, protein binding pocket, solvent, etc.). This makes it complementary to spectroscopic techniques targeting only a given molecular species such as UV-visible, fluorescence, Resonance Raman or EPR spectroscopy. In addition, time-resolved IR spectroscopy makes it possible not only to put in evidence the presence of intermediates, spectator species, products from parallel reactions and so on, but also to obtain some information on the structure of these species, even if they are short-lived. Furthermore, changes in the surrounding environment associated to the reactions (e.g., on a surface where the reactants/intermediates/products are adsorbed, or in a cofactor binding pocket of a photoactive protein) can also be followed. The advantage compared to other techniques capable of providing time-resolved structural data (e.g., time-resolved crystallography) is that minimal sample preparation is required, that data can be normally acquired in a few hours, and non-heavy data treatment is required.

Time-resolved IR absorption spectroscopy has been applied to a huge variety of chemical reactions and physical processes, ranging from combustion [1] to sol-gel processing [2] or heterogeneous catalysis [3, 4]. However, it is particularly well suited for photoinduced reactions, given the huge advantage of being able to start the reaction by light in addition to reactant mixing, heating or other approaches. Indeed, time-resolved IR absorption spectroscopy has been applied to gas-phase photochemical reactions since the early ' 80 s [5]. In addition, time-resolved IR emission spectroscopy has been largely applied [6]; however, this technique is not covered by the present review. The first applications of time-resolved IR absorption spectroscopy to photochemical reactions in liquid state [7], solid state [8], in the field of metal-organic photochemistry [9] or on biological samples [10] date back to the same decade. However, especially for photochemical reactions in biological systems, it has appeared immediately that a difference approach was required, as the tiny signals of interest related to the evolution of the reaction were hidden, in the absolute spectrum, by much more intense-and non-evolving with time- - spectral contributions from the non-reactive part of the sample. Furthermore, it was also clear that-even for slow photoreactions - the possibility of inducing the reaction in the IR spectrometer sample compartment made it possible (1) to detect smaller signals and (2) to increase the time resolution.

Several reviews on time-resolved IR spectroscopy applied to photochemical reactions in gas-phase [11], in solution [12], in solid state [13], or to photobiological reactions [14-16] are available in the literature. However, to our knowledge, no review covering all these fields exist. Even more surprisingly, reviews are seldom devoted to non-experts in time-resolved IR spectroscopy. This tutorial review aims at filling these two gaps. On the one hand, it explains, keeping the language as simple as possible, the main experimental techniques. On the other hand, it aims at showing the power of time-resolved IR spectroscopy, highlighting similarities and differences between the experiments and the obtained data in the different fields of application.

This review is organized as follows. In a first section, the different techniques are described, keeping technical details to a minimum level as our aim is to provide a complete overview of the available experimental approaches. Then, five "case studies" are presented, where time-resolved IR spectroscopy has been applied to different fields. In the last section, conclusions and perspectives in the field of timeresolved IR are presented.

\section{Methods}

For time-resolved IR spectroscopy applied to the study of photoinduced processes, a large number of different approaches are possible. Most often the techniques can be distinguished based on the covered timescale, and are classified as "ultrafast" time-resolved IR spectroscopy (sub-picosecond to nanosecond timescales) and "fast" time-resolved IR spectroscopy (from nanosecond to minute timescales).

\subsection{Ultrafast methods}

To reach time resolution in the picosecond range, pumpprobe methods based on pulsed ultrafast laser sources have to be employed. Pioneering applications of ultrafast methods in the IR are dated back from the mid-80s to early $90 \mathrm{~s}$, with the employment of picosecond lasers [17, 18]. In the following years, the development of compact and stable solid state ultrafast laser sources, in particular of Titanium:Sapphire lasers, boosted the development and application of non-linear spectroscopic techniques. At present, transient visible pump/mid-IR probe spectroscopy commonly reaches sub-picosecond time resolution, and is largely applied to study a variety of photoinduced processes in solution, in biomolecules and more recently also in the solid state $[4,9,19]$. 
In a typical pump-probe experiment, a high-energy narrow band pump pulse, whose wavelength is resonant to an electronic transition of the investigated sample, triggers the photoinduced process of interest. A second broadband less energetic probe pulse is used to monitor the time-dependent photodynamics of the system. In a pump-probe transient IR (TRIR) experiment, signals are measured in terms of the difference in absorption $(\Delta \mathrm{A})$ of the probe beam in the presence of pump excitation and in the absence of the pump, as a function of time. The absorption of the pump promotes part of the ground-state population towards an electronically excited state, with a different electronic distribution. The decreased population of the ground state determines a decreased absorption at frequencies which are characteristic for ground-state vibrational modes, inducing the appearance of negative bleaching signals in the transient spectrum. At the same time, positive signals will appear because of the induced probe absorption in the excited state. Indeed, since the electronic excitation can cause variations of bond lengths or molecular conformation, the absorption frequencies of vibrational modes can change upon photoexcitation, giving rise to excited-state absorption bands which can be upshifted or downshifted with respect to the corresponding ground-state frequency.

Experimentally, the mid-IR pulses used as probe in a TRIR experiment are obtained through difference frequency mixing (DFG) of two near-IR pulses (signal and idler) of appropriate frequency $(1200-2400 \mathrm{~nm})$ using a non-linear crystal, which most commonly is $\mathrm{AgGaS}_{2}$. In this way, a tunable output in the $3500-1000 \mathrm{~cm}^{-1}$ range is obtained. The signal and idler near-IR pulses are previously generated through a white light-seeded optical parametric amplifier (OPA), based on a $\mathrm{BBO}\left(\beta-\mathrm{BaB}_{2} \mathrm{O}_{4}\right)$ non-linear crystal [20].

The pump pulses in the visible are generated using a different non-linear optical parametric process, as for instance employing a non-collinear optical parametric amplifier (NOPA) [21-23], which provides a broadband output tunable in the visible spectral range, which can be narrowed using specific filters as to match the absorption of the sample. The polarization of the excitation pulse with respect to the IR probe pulse can be varied using a half waveplate and the intensity of the pump pulse can be attenuated to a value which depends on the experiment performed (from few tens $\mathrm{nJ}$ to a few $\mu \mathrm{J}$ ). The possibility of collecting TRIR spectra by changing the relative polarization of pump and probe beams (that can be set both parallel and perpendicular) allows to measure the transient anisotropy of the vibrational modes, that directly addresses the change in orientation of specific vibrational transition dipoles, thus providing direct information on conformational changes [24-26]. The time delay among pump and probe is introduced by sending one of the two beams through a motorized and computer-controlled translator stage (delay line). Alternative pump of the sample is realized by inserting a phase-locked chopper, operating at half the repetition rate of the main amplified laser, in the pump arm, in way to measure the change in transmission, and hence in optical density, between two consecutive shots.

After one of the beams passes the delay line, both the Visible-pump and mid-IR-probe pulses are focused and spatially overlapped at the sample position. In most cases, the probe beam is split in two arms, such as to generate a reference beam, which crosses the sample in a different position and is used to reduce the noise of the measurement. After passing through the sample, the mid-IR probe pulses are dispersed in a spectrograph and imaged onto a multiple-element Mercury Cadmium Telluride (MCT) detector. As an alternative detection scheme, the mid-IR probe pulses can be upconverted into the visible region, using a non-linear crystal, allowing to exploit sensitive and cheaper detectors such as a CCD or a silicon array device [27-30]. A schematic representation of a mid-IR pump-probe setup is reported in Fig. 1.

For the ultrafast methods, a relatively recent development concerns with the possibility of measuring 2-dimensional IR (2DIR) spectra in the excited state. In its more common application, 2DIR is used to study the evolution of systems in the electronic ground state, but the introduction of a further
Fig. 1 Schematic representation of a pump-probe setup for TRIR measurements

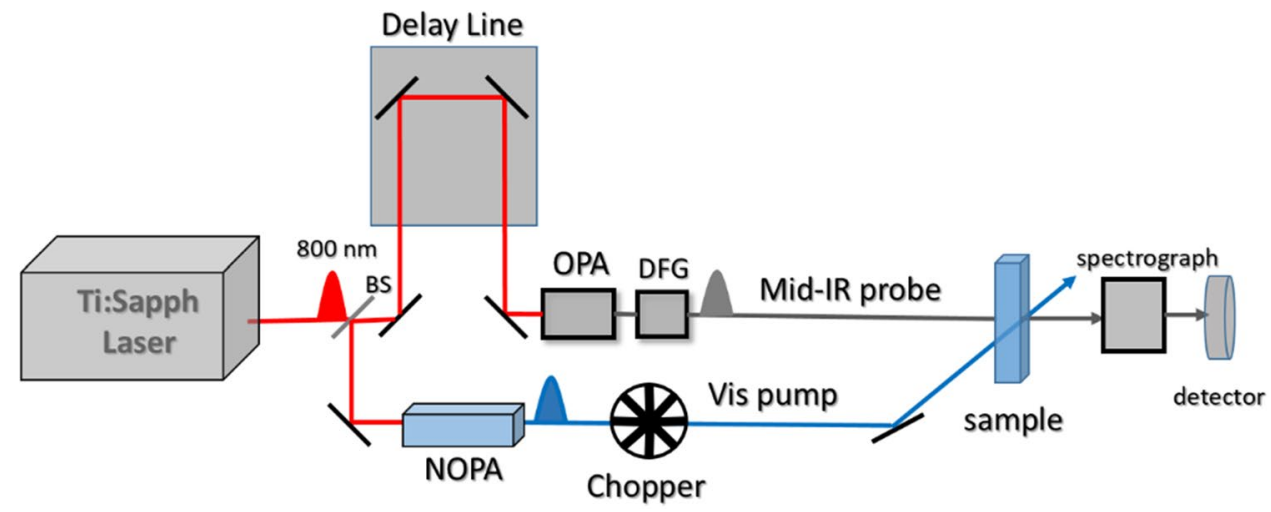


ultrashort laser pulse in the visible range, before the pulse sequence necessary to generate the 2DIR spectrum, allows recording $2 \mathrm{D}$ spectra of transient species. When referring to the ground state, the application of 2DIR spectroscopy allows to investigate the coupling between different vibrational modes and to resolve structural dynamic changes on the picosecond timescale. 2DIR is nowadays widely applied to study the dynamics of a variety of samples, including biomolecules [31-34].

The simplest way to think about the construction of a $2 \mathrm{D}$ spectrum is that of collecting a series of pump-probe spectra by scanning the frequency of the pump beam, and to stick them together such as to create a second frequency axis $[35,36]$. In the simplest situation of two coupled oscillators, absorbing at frequencies $\omega_{1}$ and $\omega_{2}$, the signals obtained in a 2DIR map will consist into two diagonal peaks, observed at frequencies corresponding to the individual energies of the two modes, and two coupling contributions, observed for excitation at $\omega_{1}$ and probing at $\omega_{2}$ and for excitation at $\omega_{2}$ and probing at $\omega_{1}$.

Experimentally, there are different ways to obtain a 2D map, all requiring the interaction of the sample with three ultrashort pulses separated by adjustable time delays. The different experimental configurations can be distinct in: (i) frequency domain mid-IR pump-probe and (ii) time domain Fourier transform experiment (three-pulse IR photon echo). For both kind of experiments, mid-IR pulses are generated by parametric and mixing processes in non-linear crystals, as described previously [31, 37-39].

The simplest implementation of frequency domain-based 2DIR measurements (pump-probe scheme) is that described above: the broadband output of one OPA is spectrally filtered using for instance a Fabry-Perot filter and used as the pump, while the broadband output of a second OPA, unfiltered, is used as probe. Multichannel detection of the probe pulse provides one frequency axis of the $2 \mathrm{D}$ spectrum, while the other axis is obtained by scanning the frequency of the IR pump pulse, adjusting the filter. The 2D spectra are obtained directly, reconstructed from TRIR slices acquired at every pump frequency.

A more compact implementation of frequency domain 2DIR relies on the pump-probe acquisition geometry of 2DIR spectra using, alternatively, an interferometer or a pulse-shaper to generate two collinear pulses. In case of the interferometric experimental scheme, the main output of one OPA is sent to a Mach-Zehnder interferometer, generating two collinear beams, used as two pump pulses [40, 41]. The two beams are directed through the sample and overlapped in space and time with the probe beam, generated through a second OPA. The relative delay between the two pump pulses $\left(t_{1}\right)$, and the delay with the probe pulse $\left(t_{2}\right)$ are scanned by motorized and computer-controlled translation stages. The probe frequency axis of the $2 \mathrm{D}$ spectrum is obtained by multichannel detection of the probe beam: in this way a frequency/time $\left(\omega_{3} / t_{1}\right)$ signal is obtained (here $\omega_{3}$ is the probe frequency and $t_{1}$ the delay among the two collinear interfering pump pulses). The obtained signal is then subject to Fast Fourier Transformation (FFT) to generate the frequency/frequency $\left(\omega_{3} / \omega_{1}\right) 2 \mathrm{D}$ spectrum. To avoid undesired artifacts (aliasing) due to an incorrect sampling of the oscillatory pattern along $t_{1}$, a HeNe laser beam is usually made copropagating with the IR pulses and its interference pattern is contextually acquired to generate an accurate timebase. Alternatively, a pulse-shaper can be used in place of the interferometer to generate the pump beam couple [42]. Pulse shaper allows fast response and the adjustment of the delay between the couple of pulses on a frequency range equal to the laser repetition rate $(1-100 \mathrm{kHz})$ [43]. Furthermore, the pulse couple generated by the pulse-shaper has an intrinsically stable and modular phase relation, allowing for the generation of pulse sequences capable to cancel scattering artifacts or to acquire the damped oscillating signal in the so-called rotating frame $[44,45]$. The construction
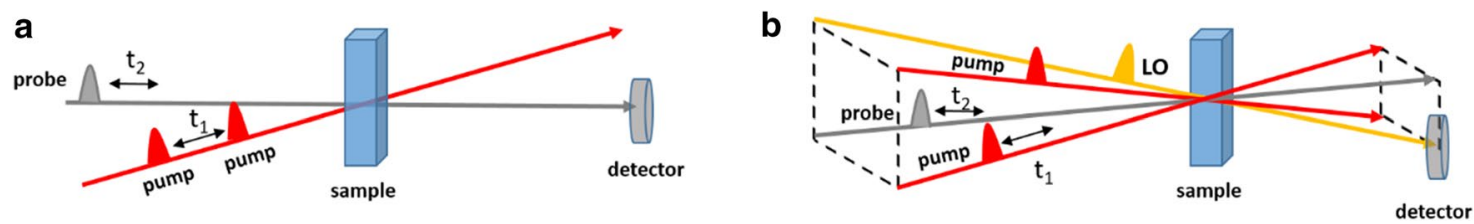

Fig. 2 Pulse sequence and geometry used to record 2D spectra in the a frequency domain and $\mathbf{b}$ time domain boxcar configuration

Fig. 3 Different pulse sequences used in transient 2DIR spectroscopy

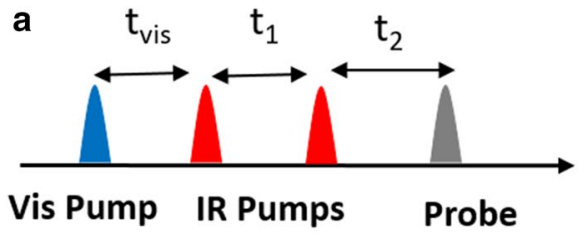

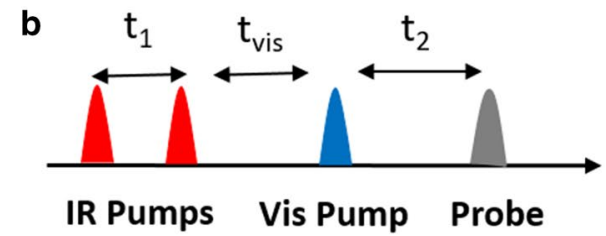


Fig. 4 Top: pulse sequence and induced processes in T-2DIR spectroscopy. Bottom: construction of T-2DIR spectrum obtained as the difference 2DIR spectra in the presence and absence of the actinic visible pulse
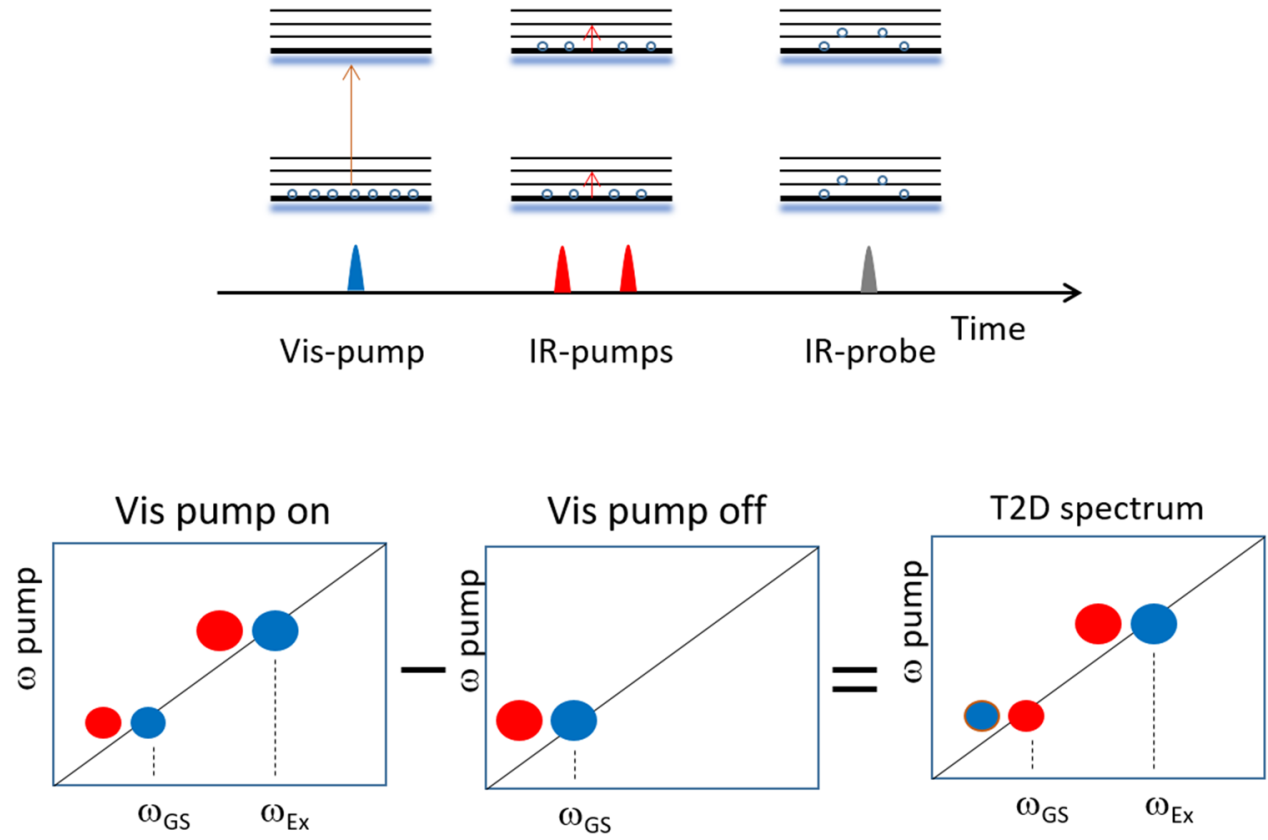

As it can be noticed in Fig. 4, T-2DIR spectra are obtained of the 2D spectrum is the same as described in case of the interferometric scheme.

In case of time domain experiments, three broadband mid-IR pulses, separated by controllable time delays, are used. The beams are arranged such as to reach the three corners of a square (boxCARS) and are overlapped and focused at the sample position, where the signal is emitted along the fourth direction of the square. To facilitate signal detection, whose intensity is generally very low, a fourth beam, termed as local oscillator (LO), is sent into the sample such as to overlap with the emitted signal and interfere with it. Fourier transformation of the oscillatory patterns created because of the superposition of signal and LO, allows to reconstruct the second frequency axis of the 2D map (the first axis is directly obtained by sending the probe beam into the spectrograph, as in pump-probe spectroscopy) [31, 38].

The different configurations are exemplified in Fig. 2.

The extension of 2DIR spectroscopy to study transient species requires the introduction of an additional visible pump pulse, which prepares the system in an excited state. Different pulse sequences can be used, as shown in Fig. 3, each giving access to a different kind of information [38, 39].

In case of the pulse sequence (a), a visible pump pulse is used to prepare a transient state or triggering a photoreaction, whose evolution is then followed by applying the 2DIR pulse sequence. The second scheme, often referred as 2DIREXSY spectroscopy, is instead useful to correlate the vibrations of a system before and after a triggered photoreaction. The expected processes and the signals obtained, following the application of the pulse sequence depicted in Fig. 3a are depicted in Fig. 4. by subtracting the 2DIR spectrum of the ground state from the 2DIR spectrum of the excited state, acquired with the visible pump on. In this way, signals pertaining to groundstate vibrations will appear with opposite sign in the double difference spectra, and new peaks, associated to excited-state vibrational modes, will be visible on both the diagonal and out diagonal regions of the map.

\subsection{Fast methods}

Fast time-resolved IR spectroscopy techniques are normally divided into three categories: those using a Michelson interferometer FTIR spectrometer, dispersive IR techniques, and those using IR lasers. However, in the last few years, new innovative instrumental approaches have been developed.

In FTIR-spectrometer-based techniques, the most straightforward approach, called rapid-scan FTIR spectroscopy, is realized by scanning the movable mirror of the interferometer as fast as possible (see Fig. 5a). Each obtained interferogram is stored and then Fourier transformed separately. In case of difference spectroscopy, a background spectrum is acquired and subtracted before the beginning of the reaction. The reaction can be triggered by a laser or lamp flash [46] (Fig. 5b, c) or using a continuous illumination source such as a lamp [47] or a LED [48] (Fig. 5d); in such case, spectra are recorded during and/or after illumination. Therefore, two different approaches are possible, as shown in Fig. 5. In the first one (Fig. 5d), spectra are recorded at increasing times after the onset of continuous illumination, to follow the time evolution of the system under constant light. Time-resolved IR spectra can be also recorded after switching off the light, 


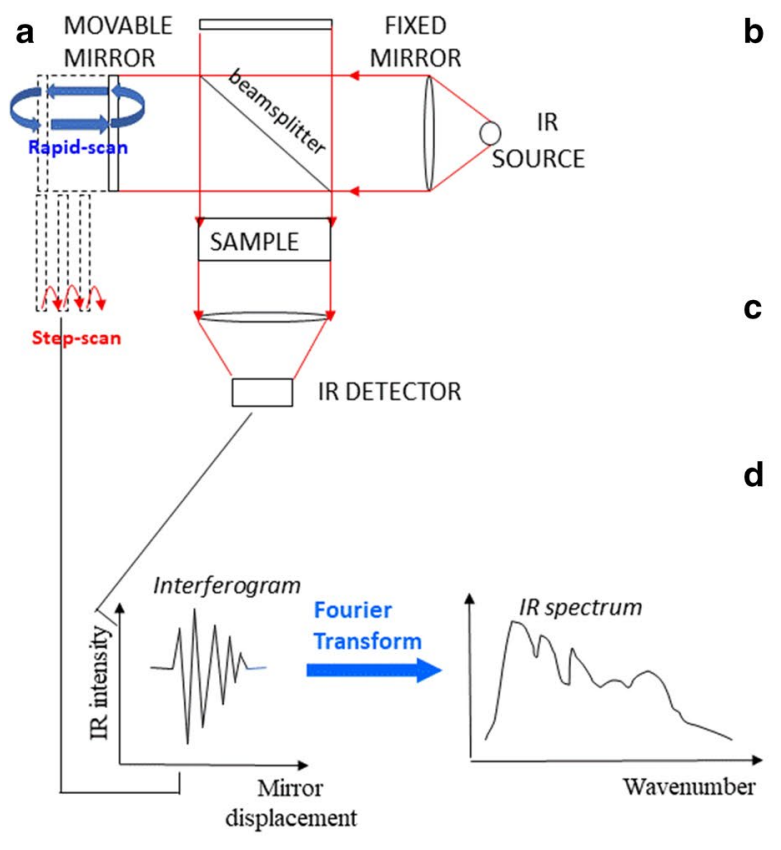

Fig. 5 Principles of time-resolved FTIR rapid-scan and step-scan. a Scheme of the Michelson interferometer-based FTIR spectrometer. In the rapid-scan mode, the movable mirror of the interferometer moves continuously at high speed: each obtained interferogram is then stored separately. In the step-scan mode, the movable mirror moves in a stepwise manner, from a stop position to another. b Timing of 1-flash-induced rapid-scan FTIR spectroscopy. The flash triggering the photoreaction arrives at the sample after the acquisition of background interferogram scans and before starting the moving mirror. To maximize the time resolution, the flash is usually synchronized with interferogram recording ("Take data" period). The delay between the flash and the beginning of the "take data" period can be set by the operator. c Timing of multiple-flash (in the figure, 3 flashes) rapidscan FTIR experiment. Flashes arrive during the intervals between interferogram recording. As in (b), usually "take data" period and flash are synchronized. d Timing of rapid-scan experiment during

to monitor the evolution of the sample in the dark after illumination, analyzing in this way the "relaxation" stage for reversible photoreactions. If a high time resolution is required by the experiment ( $\mathrm{ms}$ range), then a strict synchronization must be achieved between interferogram recording and illumination onset; in such a case, often a shutter is used [47]. In the second approach, which is by far the most applied, spectra are recorded at increasing times after a flash of light (Fig. 5b) [46]. In some special cases, spectra can be recorded also after multiple-flashes sequences or even inbetween flashes (see Fig. 5c; examples will be presented in the section dealing with time-resolved IR spectroscopy of photosynthetic reaction centers). A typical setup for flashinduced rapid-scan FTIR spectroscopy is shown in Fig. 6.
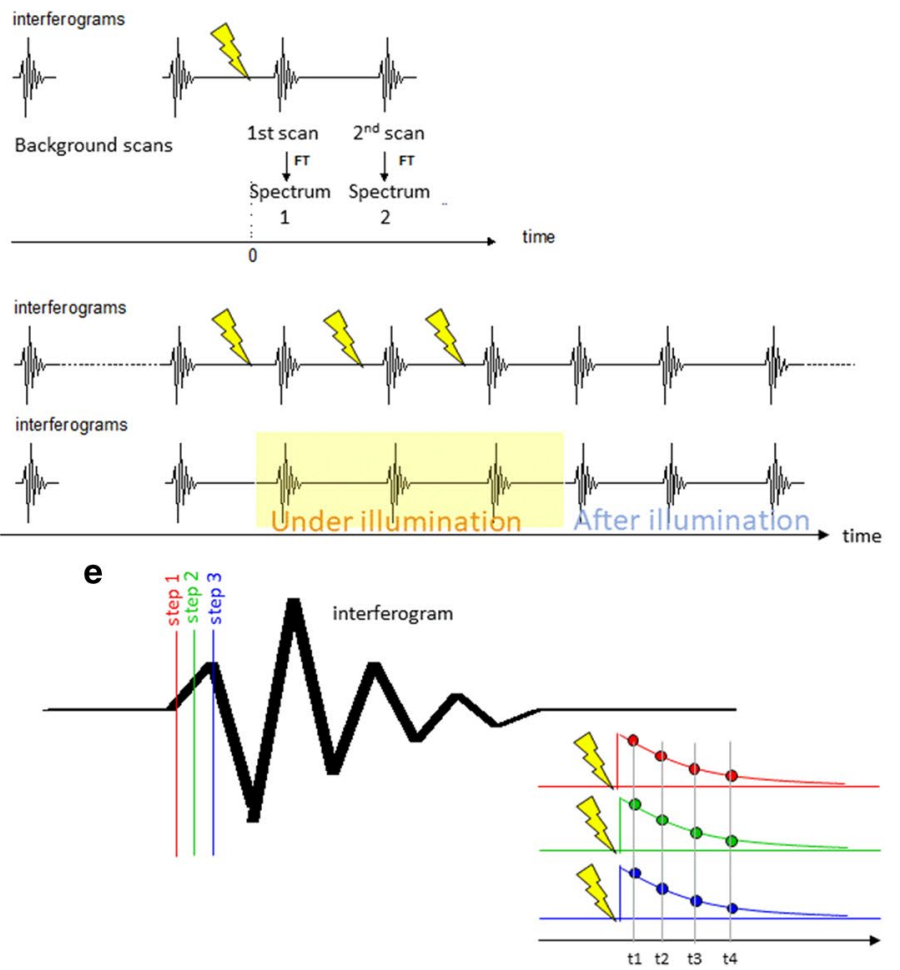

(and after) continuous illumination. After the last background scan, continuous light (Lamp, LED, etc.) is switched on. Interferograms are recorded at increasing times after the onset of light. Light switching off takes place between the recording of two interferograms. To maximize the time resolution, onset and switching off of light is synchronized with interferogram recording. Optical shutters (opening/closing times $<1 \mathrm{~ms}$ ) are often used. e Scheme of a step-scan experiment. For each stop position of the moving mirror, a light flash starts the reaction, and a transient IR signal is recorded. When a complete set of transients corresponding to a complete set of stop positions (covering the whole distance traveled by the moving mirror) is recorded and digitized, interferograms at different times can be reconstructed. For instance, all the points of each transient corresponding to time $t_{1}$ will lead to reconstruct the interferogram at time $t_{1}$ after the flash; all the points of each transient corresponding to time $t_{2}$ will lead to reconstruct the interferogram at time $t_{2}$ after the flash. And so on

Rapid-scan FTIR spectroscopy is limited in its time resolution to the millisecond timescale, because of mechanical limitations to the velocity of mirror movement. The instrument must be equipped with a fast response detector which generally consists in a liquid $\mathrm{N}_{2}$-cooled photoconductive or photovoltaic MCT detector.

In the so-called step-scan FTIR spectroscopy [49], the approach is completely different. The movable mirror of the interferometer moves in a stepwise manner (Fig. 5A, E). For each stop position, the photoreaction is started by a laser flash. Interferograms and, therefore, spectra are reconstructed from a series of transients IR signals. The maximal time resolution is in the $10-100 \mathrm{~ns}$ timescale, depending on the response time of the detector and on 

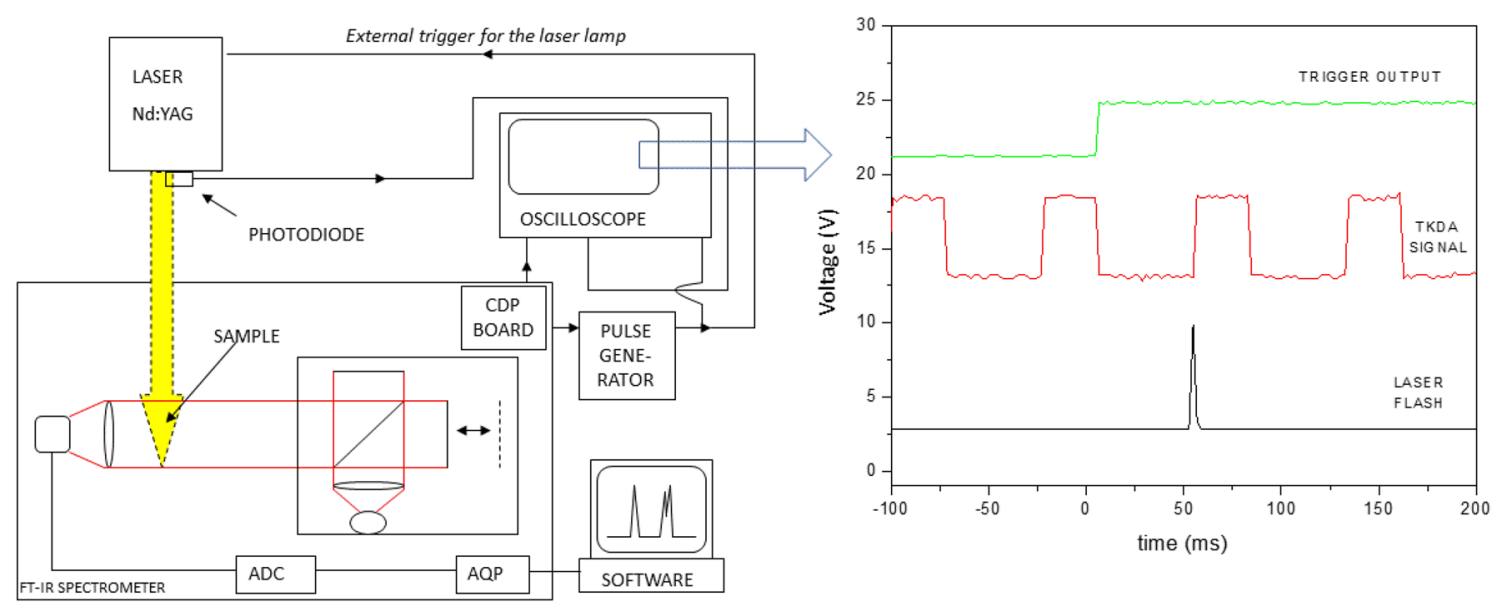

Fig. 6 Typical setup for flash-induced rapid-scan FTIR spectroscopy with ms time resolution. To realize this time resolution, synchronization between the laser starting the photoreaction, mirror movement and interferogram recording must be achieved. In the shown configuration, the master is the spectrometer CDP (Counter-Delay Phase lock looping) board which controls mirror movement and interfero-

the speed of the Analog-to-Digital Convertor (ADC). The step-scan technique can be easily applied to reversible light-induced processes, whereas irreversible processes or slow-relaxing systems require sample replacement. Other drawbacks in step-scan FTIR spectroscopy are the delicate data acquisition and data processing steps (to avoid nagging artifacts) [50], and the possibility of unwanted signals arising from photothermal effects, which should be eliminated or minimized by temperature control and/ or adequate setting of the laser power [51].

Other FTIR-based techniques have been applied in the past (ultrarapid scanning [52, 53], stroboscopic FTIR [54], and others) but they are seldom used nowadays and will not be described here. It should be mentioned that interesting results can be obtained also by conventional FTIR spectrometers, non-equipped with rapid-scan/step-scan option, if a time resolution in the second/minute timescale is sufficient to monitor the reaction under study.

Dispersive IR instruments avoid the limitations and complications of rapid-scan and step-scan FTIR spectroscopy. They can either use a monochromator [55], or an array of detectors [56]. Recently, new dispersive instruments showing a strongly enhanced signal-to-noise ratio have been developed [57], and are commercially available.

A completely different approach relies on the use of IR lasers. Despite their use dates back to more than 30 years ago [58], the relevance of this approach increased enormously in the last $\sim 10$ years thanks to the availability of tunable quantum cascade lasers (QCLs). QCLs have been invented in 1994. They are electrically pumped unipolar semiconductor lasers, emitting light through "intersub-band" optical gram recording ("Take data" or "TKDA" signal). The pulse generator makes it possible to introduce a suitable time delay between the signal coming from the CDP board (synchronized with mirror movement) and the input for laser firing, so that the laser can be fired at a fixed time $(1,2,3 \ldots . \mathrm{ms})$ before the beginning of the "Take data". Visualization of experiment timing on an oscilloscope is mandatory

transitions, in contrast to bipolar semiconductor lasers being "interband" lasers. As the photon energy resulting from intersub-band transitions is independent of the band gap of the material in the active region, it can be tuned by adapting the so-called quantum well thickness [59, 60]. As recently demonstrated by Schnee et al., QCLs can be easily coupled with standard rapid-scan FTIR spectrometers, by directing the QCL beam through the spectrometer towards the sample compartment, but without crossing the interferometer. This can be achieved by properly adapting the mirrors arrangement [61]. Optical benches containing four QCLs emitting in different but slightly overlapping wavenumber ranges within $1876-905 \mathrm{~cm}^{-1}$ are commercially available. Within the latter ranges, each of the QCLs is tunable to one single chosen wavenumber (with an accuracy $\leq 1 \mathrm{~cm}^{-1}$ ). Provided that the spectrometer is equipped with an appropriate MCT detector, a setup in which such a QCLs bench is coupled with a standard FTIR spectrometer allows easily switching from full standard rapid-scan FTIR measurements over a broad range of wavenumbers $\left(6000-650 \mathrm{~cm}^{-1}\right)$, but limited to milliseconds time resolution, to fast QCL diagnostic at one of the wavenumbers of interest [61]. This typically allows measuring the temporal evolution of one single species among others within an interesting spectral region which contains several molecular/structural fingerprints. The QCLirradiation mode reaches a time resolution that depends only on the response time of the detector (typically $50 \mathrm{MHz}$ ), the QCL pulse rate (settable up to $1 \mathrm{MHz}$ ), and the data acquisition system (which limited the time resolution of Schnee et al.'s setup to $4 \mu \mathrm{s}$ ) [61]. Therefore, while still providing the whole qualitative and quantitative information for which 
rapid-scan FTIR spectrometers are generally used, a combined QCL-FTIR spectrometer drastically pushes the limits in monitoring dynamic phenomena, with a large operating flexibility [61].

Recently, also pump-probe measurements (until 2012 limited to the fs-ps range) have been extended to the millisecond timescale, employing methods based on the synchronization of two ultrafast laser sources [62-64].

The most impressive technical advancements in timeresolved IR difference spectroscopy, however, are even more recent. Dual-comb time-resolved IR spectroscopy makes it possible to perform single-shot experiments to obtain timeresolved spectra in $\mathrm{a} \sim 60 \mathrm{~cm}^{-1}$ spectral region with a time resolution of $320 \mathrm{~ns}$ [65]. Finally, a dispersive IR setup based on a Féry Spectrometer and on a IR synchrotron source have been developed, making possible to obtain single-shot spectra in the $1800-1100 \mathrm{~cm}^{-1}$ spectral region [66].

\subsection{Sample preparation and data analysis}

Compared to visible pump-probe spectroscopy, sample handling and preparation in case of mid-IR transient measurements require some specific care. In case of liquid samples, not all solvents are suitable, and attention should be paid to avoid solvents presenting intense vibrational bands in the region of interest for the sample. In the majority of cases, cells with short path lengths on the order of $100 \mu \mathrm{m}$ or less are used, which helps in decreasing problems caused by background solvent absorption. Water has a very high absorption cross-section in the midIR, so water-dissolved samples are generally prepared as very thin films. Alternatively, deuterated water can be used to shift the solvent absorption in a different spectral region. Liquid samples are generally prepared by squeezing a few drops of solution among two calcium fluoride windows, separated by a thin Teflon spacer to reach the desired path length. Attention should be paid to avoid the formation of bubbles or heterogeneous zones in the samples, which could increase scattering and noise. Sample concentration, in the milli-molar range, is much higher than those usually required for measurements in the visible region, because of the small absorption cross-section of vibrational bands, which can be a problem in some cases, particularly with biomolecules. Photodegradation of the sample can be minimized by mounting the sample cell on motorized holders, or using a flow cell in case the sample quantity allows for it. The use of motorized cell holders also allows to maintain fresh sample conditions during repetitive measurements, necessary because in most cases signals are very small and data accumulation is required to achieve a good signal-to-noise ratio. Solid-state samples are more difficult to handle, mainly because of problems related to heterogeneities or little transparency. In case of measurements performed in transmission mode, solid state samples are prepared as thin films and deposited on IR transparent windows, mostly through spin-coating techniques. Finally, attention should be paid to avoid artifacts related to the use of high-power density, which can cause excited-state annihilation or increase the local heat of the sample, inducing distortions on both the kinetic traces and the spectral shape. To reduce the absorption of water vapor present in the atmosphere, TRIR setups are inserted in closed boxes purged with dry nitrogen. In time-resolved FTIR spectrometers, especially when operating in the stepscan mode, vacuum technologies are also widely used.

For time-resolved IR spectroscopy of solid adsorbers/ catalysts under an adsorbent/reactive gaseous atmosphere (see case study on photocatalysis in Sect. 3.2), the solid samples are generally pelletized. If it is not possible to obtain a thin and homogeneous pellet of a given material, the latter can also be impregnated (typically in water/alcohol) onto a silicon or $\mathrm{CaF}_{2}$ plate. The water/alcohol will be evacuated in situ upon starting the experiment. The latter way of sample preparation generally allows only qualitative measurements, however. The auto-supported pellets or plate-supported samples are then introduced in the IR cell thanks to an appropriate sample holder. In vacuum setups, evacuating the IR cell and introducing the desired pressure of reactant must be done smoothly to avoid detaching and breaking the pellet/plate. In continuous flow setups dedicated to operando photocatalytic studies, the pellet/plate is typically placed into a "sandwich" transmission IR reactor-cell equipped with $\mathrm{KBr}$ windows at each side of the sample holder (to reduce the dead volume) and with a heating system [67]. The outlet gas flow can be analyzed in an annex optical bench containing a dedicated gas-IR cell, or in a mass spectrometer or gas chromatograph. In all cases, prior to measurements, the IR cell must be carefully checked for leaks.

Several strategies have been proposed to increase the signal-to-noise ratio, to analyze the time-resolved IR spectra disentangling spectral contributions from different species, or to interpret IR signals (especially from transient states). A detailed description of these methods is beyond the scope of this tutorial review; it should, however, be mentioned that in data treatment several approaches are possible, either relying on kinetic models (so-called hard modeling approaches [68]) or keeping the analysis free from constraints (so-called softmodeling approaches [69]). Mixed hard-soft approaches have also been developed [70], as well as more tailored strategies [71]. 2D correlation spectroscopy is also often used to establish the concomitance or non-concomitance of different events [72].

In IR spectroscopy, band attribution is carried out by performing experiments in different solvents (e.g., $\mathrm{H}_{2} \mathrm{O}$ and $\mathrm{D}_{2} \mathrm{O}$ ) or using isotopically labeled molecules. For molecules in complex environments (cofactors or pigments in 
protein binding pockets, reactant adsorbed on surfaces, etc.), comparison with the IR spectra of the studied molecules in well-known environments (cryogenic matrices, solvents, etc.) is also a common strategy. Theoretical calculations (Density Functional Theory-DFT, Quantum-Mechanics/ Molecular Mechanics-QM/MM, Normal mode analysis NMA, etc.) are also routinely used, and often coupled to the experimental above-mentioned strategies. This approach (comparison with molecules in well-characterized environment, often associated with calculations) holds true also for time-resolved IR spectroscopy, and it makes possible to assign bands of reactants, products, by-products, etc. However, this approach may become particularly difficult for IR bands of putative transient species (excited states, shortlived radicals, etc.), as these species are often difficult to obtain in separate experiments, so that a direct comparison is not possible. In these cases, the theoretical approaches are basically the only way to achieve band attribution. DFT (see for instance Mezzetti et al. [73]), QM/MM (see for instance Rohani et al. [74]) or more advanced calculations (Macaluso et al. [75]; Petrone et al. [76]) become particularly useful.

\section{Case studies}

\subsection{Photosynthetic reaction centers}

\subsubsection{Bacterial reaction centers}

Time-resolved IR spectroscopy has been widely applied to photobiological reactions since the mid ' 80 s, [14, 15] and in particular to isolated photosynthetic protein complexes since 1990 [19, 77]. In the following, we will not consider results obtained on light harvesting processes [19] or photoprotective mechanisms $[48,73,78]$. We will instead describe results obtained on photosynthetic reaction centers (RCs), where the real photochemistry of photosynthesis takes place. According to their internal photochemistry, photosynthetic RCs are normally divided into type I and type II RCs. We will focus here on type II RCs, and in particular on the two most studied systems: the type II bacterial RCs and photosystem II. The interested reader can refer to recent reviews describing the most interesting results obtained on photosystem I and other type I photosynthetic RCs [79, 80]. Especially for photochemical reactions in biological systems, it has appeared immediately that a difference approach was required, as the tiny signals of interest related to the evolution of the reaction were hidden, in the absolute spectrum, by much more intense-and non-evolving with time- - spectral contributions from the non-reactive part of the sample. The type II bacterial RC, especially from $R$. sphaeroides, an anoxygenic purple photosynthetic bacterium, has played a key role in clarifying structure-function relationships of primary photosynthetic reactions. As in the other RCs, a series of cofactors are involved in the ultrafast conversion of an excited state $\mathrm{D}^{*} \mathrm{~A}$ to a charge-separated state $\mathrm{D}^{+} \mathrm{A}^{-}$(D stands for electron Donor, A for electron Acceptor). In R. sphaeroides RC, the primary event is the photooxidation of the so-called primary donor $\mathrm{P}_{870}$ (a dimer of Bacteriochlorophyll $a$ molecules) by electron transfer to a first acceptor $\mathrm{H}_{\mathrm{A}}$, a pheophytin. On slower timescales, $\mathrm{P}_{870}{ }^{+}$is reduced back to $\mathrm{P}_{870}$ by a cytochrome, whereas the electron of the primary acceptor is transferred subsequently to two quinones, $Q_{A}$ and $\mathrm{Q}_{\mathrm{B}}$. The photoreaction is repeated so that a double reduction and double protonation of $\mathrm{Q}_{\mathrm{B}}$ is achieved. The formed $\mathrm{Q}_{\mathrm{B}} \mathrm{H}_{2}$ leaves the RC. The first time-resolved IR data in isolated photosynthetic proteins were obtained on this RC [18, 81-84]. Several IR marker bands have been identified by static IR difference spectroscopy for cofactors, notably the Bacteriochlorophyll $a(\mathrm{BChl} a)$ dimer $\mathrm{P}_{870}$ and the primary and secondary quinone electron acceptor $\mathrm{Q}_{\mathrm{A}}$ and $\mathrm{Q}_{\mathrm{B}}$, in oxidized or reduced states [85]. In addition, IR bands for amino acid side chains $[86,87]$ and internal water molecules [88] have been identified. This has made it possible to give a deep interpretation of time-resolved IR data and prompted a series of subsequent studies. A first advantage of time-resolved IR spectroscopy emerges immediately, as IR bands of each of the above-mentioned molecules are narrow and often well separated from others. On the one hand, the difference approach makes it possible to visualize only the molecular groups undergoing changes; on the other hand, the similar (or same) chemical structure of different reactants, intermediates or products is in most cases not a major problem, as IR bands are strongly influenced by the environment and, therefore, site-specific. For instance, semiquinones $\mathrm{Q}_{\mathrm{A}}{ }^{-}$and $\mathrm{Q}_{\mathrm{B}}{ }^{-}$, despite being the same chemical species-a radical anion of ubiquinone-10-have specific well-separated marker bands (at 1467 and $1479 \mathrm{~cm}^{-1}$, respectively).

The primary charge separation $\mathrm{P}^{+} \mathrm{H}_{\mathrm{A}}{ }^{-}$and the subsequent electron transfer to $\mathrm{Q}_{\mathrm{A}}$ have been studied in a series of ultrafast IR studies on wild-type RCs [84, 89], mutant RCs [90-92], and chemically modified RCs [93].

A much livelier scientific debate involved the electron transfer between the two quinones $\mathrm{Q}_{\mathrm{A}}{ }^{-} \mathrm{Q}_{\mathrm{B}} \rightarrow \mathrm{Q}_{\mathrm{A}} \mathrm{Q}_{\mathrm{B}}{ }^{-}$. A major breakthrough was the observation, by single wavenumber time-resolved IR, that the electron transfer reaction is coupled to the protonation of the side chain of a residue close to the $\mathrm{Q}_{\mathrm{B}}$ binding pocket, Glu-L212 [86]. It should be pointed out that time-resolved IR spectroscopy makes it possible to follow both electron transfer and proton transfer reactions, a piece of information almost impossible to get from other spectroscopic techniques. In mutant RCs where the electron transfer is slowed down, the protonation of Glu-L212 was found to be concomitant with the electron transfer (Fig. 7) 

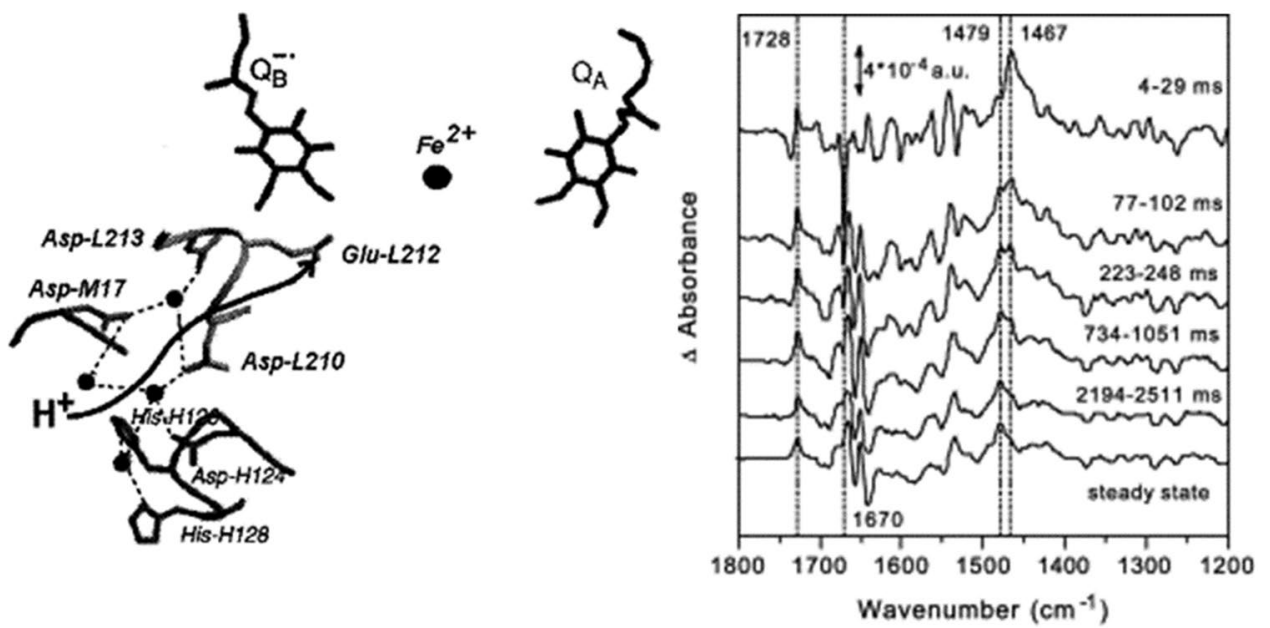

Fig. 7 Rapid-scan FTIR difference spectra recorded after one flash on the $\mathrm{DN}(\mathrm{L} 210) / \mathrm{DN}(\mathrm{M} 17)$ double mutant RC from $R$. sphaeroides. As shown in the left picture, in wild-type RCs, the protonable side chain of two D (Asp) amino acid are involved in a proton transfer pathway. Upon double mutation to $\mathrm{N}$ (Asn), the proton transfer pathway is perturbed. The double mutation slows down the proton transfer from the cytoplasm towards the $\mathrm{Q}_{\mathrm{B}}$ site, and the electron transfer between $\mathrm{Q}_{\mathrm{A}}$ and $\mathrm{Q}_{\mathrm{B}}$, making the overall reaction accessible to rapid-scan FTIR. The use of external donor ensured fast reduction $(<<4 \mathrm{~ms})$ of the oxidized primary donor $\mathrm{P}_{870}{ }^{+}$, eliminating thereby its very intense IR bands. Electron transfer could be followed through previously identified marker bands at $1467 \mathrm{~cm}^{-1}\left(\mathrm{Q}_{\mathrm{A}}^{-}\right)$and $1479 \mathrm{~cm}^{-1}\left(\mathrm{Q}_{\mathrm{B}}{ }^{-}\right)$. The protonation of the side chain of Glu-L212 could be followed through

the previously identified marker band at $1728 \mathrm{~cm}^{-1}$. A unique piece of information deduced from these time-resolved FTIR data was that beside proton uptake and electron transfer, also protonation of the Glu-L212 side chain is slowed down by the double mutation. Rapid-scan FTIR experiments on wild-type RCs (and other mutants) strongly suggest that the slow protonation of Glu-L212 and slow electron transfer between the quinones are coupled processes in native RCs [46]. Figure above has been reprinted from Biochimica et Biophysica Acta 1553, A. Mezzetti, E.. Nabedryk, J. Breton, MY Okamura, ML Paddock, G. Giacometti, W. Leibl, "Rapid-scan Fourier transform infrared spectroscopy shows coupling of Glu-L212 protonation and electron transfer to $\mathrm{Q}_{\mathrm{B}}$ in Rhodobacter sphaeroides reaction centers" Copyright 2002, with permission from Elsevier

[46]. Furthermore, electron and proton transfer reactions could be followed in the same series of time-resolved FTIR difference spectra, i.e., simultaneously. However, interpretation of time-resolved IR difference spectroscopy data, especially those obtained through step-scan FTIR, which requires a large number of actinic events, should also be done with care. In two following papers, the Gerwert group suggested that $\mathrm{Q}_{\mathrm{B}}{ }^{-}$formation precedes $\mathrm{Q}_{\mathrm{A}}{ }^{-}$oxidation, and proposed the existence of an intermediate $\mathrm{X}$, electron donor to $\mathrm{Q}_{\mathrm{B}}$ $[94,95]$. This hypothesis was quite revolutionary, as no other techniques suggested indications for such an intermediate. In an elegant paper using static FTIR difference spectroscopy [96], Breton showed that the $\mathrm{Q}_{\mathrm{A}}{ }^{-} \mathrm{X}^{\mathrm{ox}} / \mathrm{Q}_{\mathrm{A}} \mathrm{X}$ difference spectrum reported in the work of Hermes et al. [95] is essentially equivalent to a $\mathrm{Q}_{\mathrm{A}}{ }^{-} \mathrm{Q}_{\mathrm{B}} / \mathrm{Q}_{\mathrm{A}} \mathrm{Q}_{\mathrm{B}}{ }^{-}$double difference spectrum constructed from the pure $\mathrm{Q}_{\mathrm{A}}{ }^{-} / \mathrm{Q}_{\mathrm{A}}$ and $\mathrm{Q}_{\mathrm{B}}{ }^{-} / \mathrm{Q}_{\mathrm{B}}$ FTIR difference spectra, demonstrating as highly unlikely the existence of such an intermediate X. Most probably, sample degradation occurring during the time-resolved FTIR experiments reported in references $[94,95]$ (carried out mainly by stepscan FTIR, not well suited for photoreactions slowly recovering to the initial state) somehow did not allow a proper analysis of time-resolved data.

Conversely, several time-resolved IR papers suggest the existence of a transient proton acceptor, probably the side

chain of an Asp residue, in the proton transfer channel, located mid-way between the proton entry point and the $\mathrm{Q}_{B}$ pocket $[86,97,98]$. In this case, the power of time-resolved IR difference spectroscopy is even more evident, as it has not only put in evidence the time evolution of proton transfer, but it was also able to "catch" a transient proton acceptor.

Time-resolved FTIR spectroscopy was also used to study the $\mathrm{Q}_{\mathrm{A}}{ }^{-} \mathrm{Q}_{\mathrm{B}}{ }^{-} \rightarrow \mathrm{Q}_{\mathrm{A}} \mathrm{Q}_{\mathrm{B}}{ }^{2-}$ reaction and the ubiquinol $\mathrm{Q}_{\mathrm{B}} \mathrm{H}_{2}$ release. Mezzetti and Leibl in 2005 studied by rapidscan FTIR technique this process in wild-type isolated RCs [97]. Rapid-scan FTIR difference spectra recorded after two flashes showed indication for the displacement of $\mathrm{Q}_{\mathrm{B}} \mathrm{H}_{2}$ from its binding pocket towards the outside. A key point to follow the process was the synchronization between interferogram recording and laser firing, so that precise and known delays were inserted between the flash (both the 1st and the 2nd) and the beginning of interferogram recording. This delay (4 ms) was necessary to let external donors to reduce the oxidized Primary donor $\mathrm{P}_{870}{ }^{+}$, thereby making it possible to repeat a second lightinduced charge separation and, eventually, to obtain a double reduction of the $\mathrm{Q}_{\mathrm{B}}$ cofactor.

The process was also studied by rapid-scan FTIR spectroscopy under and after continuous illumination [97], exploiting also chemometric tools and 2D correlation 
Fig. 8 a Structure of the $\mathrm{Mn}_{4} \mathrm{CaO}_{5}$ cluster and of the surrounding water molecules and amino acid residues. The structure was depicted using the coordinates of Protein Data Bank entry 4UB6.58 [113]. b S-State cycle leading to photoinduced water oxidation. $\mathbf{C}$ Time courses of $\Delta \mathrm{A}$ at $1514 \mathrm{~cm}^{-1}$ (green line, marker band for $\left.\mathrm{Y}_{\mathrm{Z}^{*}}\right), 1400 \mathrm{~cm}^{-1}$ (blue line, marker band for $\mathrm{COO}^{-}$), and 1256 (red line, marker band for $\mathrm{Y}_{\mathrm{Z}}$ ) $\mathrm{cm}^{-1}$ in the $\mathrm{S}_{2} \rightarrow \mathrm{S}_{3}$ transitions in PSII core complexes at a pH 5.0, b pH 6.0, $\mathbf{c}$ pH 7.0, and $\mathbf{d} \mathrm{pH}$ 8.0. Black lines are simulated traces obtained by global fitting of the three experimental traces at each $\mathrm{pH}$. The scale bars indicate a $\Delta \mathrm{A}$ of $5 \times 10^{-5}$. Adapted with permission from Takemoto et al. [110]. Copyright 2019 American Chemical Society a

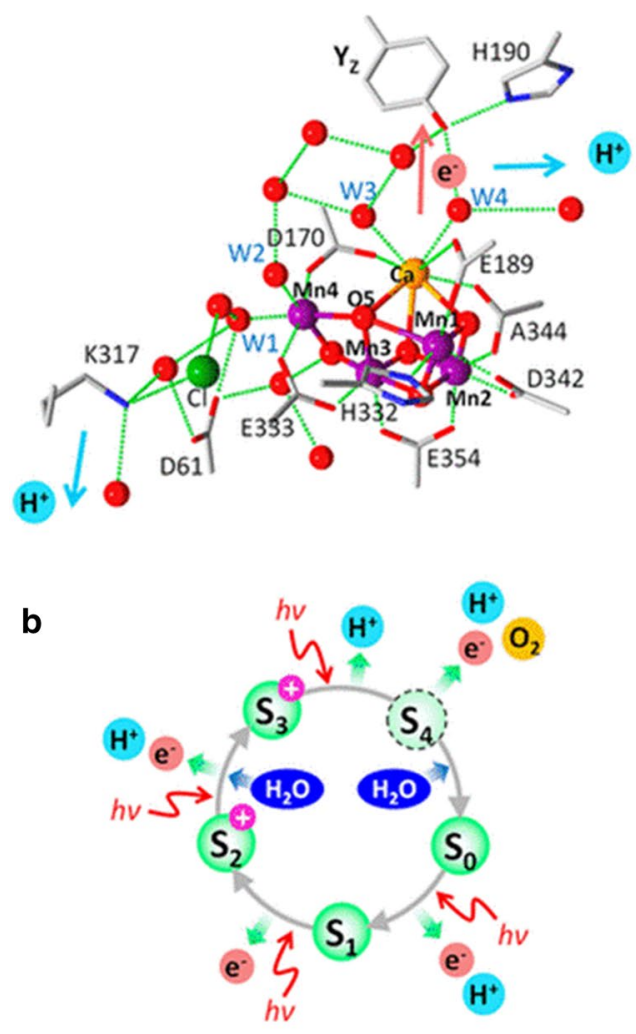

C

c $\quad \mathrm{S}_{2} \rightarrow \mathrm{S}_{3}$

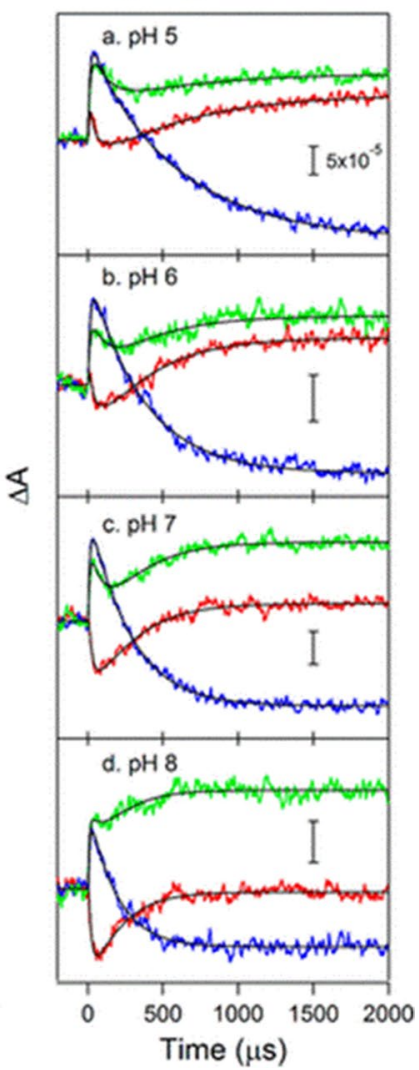

analysis for an in-depth understanding of the mechanism [72].

The process of ubiquinol release was also studied by time-resolved FTIR under and after continuous illumination in bacterial membranes, a more intact environment, and therefore, a more appropriate condition to investigate $\mathrm{Q}_{\mathrm{B}} \mathrm{H}_{2}$ release [47, 69, 70, 99].

It should be noticed that whereas monoelectronic reduction of $\mathrm{Q}_{\mathrm{B}}$ can in principle be followed by other spectroscopic techniques (e.g., UV-Vis, or EPR) this is much more difficult for $\mathrm{Q}_{\mathrm{B}} \mathrm{H}_{2}$ formation and nearly impossible for $\mathrm{Q}_{\mathrm{B}} \mathrm{H}_{2}$ release (corresponding to a movement from a protein binding pocket to the outside of the protein).

\subsubsection{Photosystem II reaction centers}

Time-resolved IR spectroscopy has been extensively applied to the RC of Photosystem II (PSII) from plants and cyanobacteria. The architecture of the RC of PSII resembles that of the RC of purple bacteria. A primary donor called $\mathrm{P}_{680}$ absorbs light and gets photooxidised to $\mathrm{P}_{680}{ }^{+}$, mimicking what happens in bacterial RCs for $\mathrm{P}_{870}$. On the so-called acceptor side ("acceptor" because it accepts the electron coming from $\mathrm{P}_{680}$ ), the $\mathrm{Q}_{\mathrm{A}} / \mathrm{Q}_{\mathrm{B}}$ electron acceptor groups are present, with the same role as in the above-described bacterial RC. PSII also contains on the donor side a redoxactive tyrosine residue, $\mathrm{Y}_{\mathrm{Z}}$, acting as an electron donor, and a $\mathrm{Mn}_{4} \mathrm{CaO}_{5}$ water oxidizing complex (WOC), as shown in Fig. 8a. At the WOC, two $\mathrm{H}_{2} \mathrm{O}$ molecules are oxidized to give a $\mathrm{O}_{2}$ molecule and four $\mathrm{H}^{+}$. This $2 \mathrm{H} 2 \mathrm{O} \rightarrow \mathrm{O} 2+4$ $\mathrm{H}++4 \mathrm{e}-$ reaction takes place through 5 intermediate states called $\mathrm{S}_{i}(i=0-4)$ : the higher the $\mathrm{i}$ value, the higher the oxidation state of the $\mathrm{MnCaO}_{5}$ cluster (see Fig. 8b). Each step of electron extraction (corresponding to a $\mathrm{S}_{i} \rightarrow \mathrm{S}_{i+1}$ transition) is determined by the need of re-reducing the $\mathrm{Y}_{\mathrm{Z}}$. radical, and therefore, indirectly also re-reducing the oxidized form of primary donor, $\mathrm{P}_{680}{ }^{+}$to $\mathrm{P}_{680}$. The $\mathrm{S}_{4}$ state is a transient state, because it immediately releases $\mathrm{O}_{2}$ and relaxes back to the $S_{0}$ state.

Therefore, PSII couples the two-electron reduction of $\mathrm{Q}_{\mathrm{B}}$ (in the acceptor side) to a four-electron oxidation of the $\mathrm{MnCaO}_{5}$ cluster (in the donor side).

As for bacterial RCs, ultrafast IR has been used to investigate the initial process of photoinduced charge separation [100-102]. However, most time-resolved IR studies have been-and still are-focused on understanding the watersplitting chemistry of PSII.

With the aim of better understanding the different steps of water oxidation, in 2001 Noguchi and Sugiura used a multiple-flash approach coupled to time-resolved FTIR with 
a time resolution of $10 \mathrm{~s}$ [103]. In this way, they were able to obtain consecutive FTIR difference spectra reflecting the $\mathrm{S}_{0} \rightarrow \mathrm{S}_{1} \rightarrow \mathrm{S}_{2} \rightarrow \mathrm{S}_{3}\left(\rightarrow \mathrm{S}_{4}\right) \rightarrow \mathrm{S}_{0}$ transitions. A similar approach was reported in the same year by Hillier and Babcock [104]. Since then, the approach has been used several times, including the low-frequency region $\left(670-350 \mathrm{~cm}^{-1}\right)$ [105]. These studies are "time-resolved" in the sense that they require a synchronization of interferogram recording with the firing of multiple flashes. They made it possible to record "static" FTIR difference spectra (reflecting all the molecular changes in the WOC) corresponding to each $\mathrm{S}_{\mathrm{i}} \rightarrow \mathrm{S}_{i+1}$ transition.

Since 2012, in a series of papers Noguchi and co-workers used time-resolved IR with a dispersive IR spectrometer to investigate the $\mathrm{S}_{0} \rightarrow \mathrm{S}_{1} \rightarrow \mathrm{S}_{2} \rightarrow \mathrm{S}_{3}\left(\rightarrow \mathrm{S}_{4}\right) \rightarrow \mathrm{S}_{0}$ transitions in wild-type and mutant PSII RCs (Fig. 8B) [55, 106-109]. Upon flash excitation, they monitored IR changes at 1400 and $2500 \mathrm{~cm}^{-1}$ which represent, respectively, the reaction (or interaction changes) of $\mathrm{COO}^{-}$groups and the changes in proton polarizability of strong hydrogen bonds. It should be noticed that no other spectroscopic techniques can follow directly changes in $\mathrm{COO}^{-}$status and proton movement.

The $S_{2} \rightarrow S_{3}$ transition was studied in details [110, 111], also by another research group [112]. In particular, results from the Noguchi group [107] suggested that during the $\mathrm{S}_{2} \rightarrow \mathrm{S}_{3}$ transition proton release is a rate-limiting step in the proton-coupled electron transfer. The authors could follow the electron transfer to $\mathrm{Y}_{\mathrm{Z}}$ thanks to IR marker bands for $\mathrm{Y}_{\mathrm{Z}} \cdot\left(1514 \mathrm{~cm}^{-1}\right)$ and for $\mathrm{Y}_{\mathrm{Z}}\left(1256 \mathrm{~cm}^{-1}\right)$. The coupling with proton transfer reactions is suggested by the $\mathrm{pH}$ dependence of $\mathrm{Y}_{\mathrm{Z}}$. reduction (see Fig. 8C). This example shows how the availability of IR marker bands for $\mathrm{Y}_{\mathrm{Z}} / \mathrm{Y}_{\mathrm{Z}}$ (involved in the photoinduced electron transfer from WOC to $\mathrm{P}_{680}{ }^{+}$), associated with a study of the $\mathrm{pH}$ dependence of the IR transients, can make it possible to determine the coupling between electron and proton transfers

The acceptor side was also the object of investigation; Nozawa and Noguchi studied by rapid-scan FTIR, the $\mathrm{pH}$ dependence of the relaxation rate of $\mathrm{Q}_{\mathrm{B}}{ }^{-}$[114]. This is an important piece of information that suggested to the authors the hypothesis that the $\mathrm{pH}$-dependent $\mathrm{Q}_{\mathrm{B}}{ }^{-}$relaxation is one of the mechanisms to regulate electron flow in PSII for its photoprotection. It is important to notice that only the use of rapid-scan FTIR made it possible to follow directly the time evolution of $\mathrm{Q}_{\mathrm{B}}{ }^{-}$through a specific $\mathrm{Q}_{\mathrm{B}}{ }^{-}$IR marker band.

Rapid-scan FTIR was also used in DCMU-treated PSII to investigate the effect of multiple flashes in building-up a « light-adapted state» [115], as already observed in bacterial RCs [88]. Indeed, $S_{2} Q_{A}{ }^{-} / S_{1} Q_{A}$ difference spectra recorded after a series of 20 saturating flashes showed a threefold decreased rate of charge recombination compared to timeresolved $\mathrm{S}_{2} \mathrm{Q}_{\mathrm{A}}{ }^{-} / \mathrm{S}_{1} \mathrm{Q}_{\mathrm{A}}$ difference spectra recorded after a single saturating flash. The authors proposed that dielectric relaxation processes inside the RC play a key role. In this case, not only semiquinone re-oxidation, but also the recovery of the $S_{1}$ state from the $S_{2}$ state were followed directly through marker IR bands.

Time-resolved IR was also coupled to measurement methods different from transmission. An interesting example is the use of Rapid-scan FTIR in the Attenuated Total Reflectance (ATR) mode. It was recently used to investigate the photoassembly of the $\mathrm{Mn}_{4} \mathrm{CaO}_{5}$ cluster in PSII (a process called photoactivation) [116]. Flash-induced, rapidscan FTIR difference spectra of the protein complex upon binding of $\mathrm{Mn}^{2+}$ were obtained, shedding some light on the chemistry of the process. This approach shows that more complex photochemical processes, involving ligands that are "external" to the photoactive protein, can be followed. Indeed, it is expected that, in the future, photobiological time-resolved IR studies will be performed more and more often in the ATR mode.

\subsection{Gas-phase photocatalysis}

Time-resolved IR absorption spectroscopy can also be applied to photoinduced reactions within a gas phase [117, 118], or at a gas-solid interface, typically on a heterogeneous photocatalyst [119-123]. The present section of the review focuses on the latter case, where time-resolved IR is a unique way to directly monitor the interactions between adsorbed molecules and the photocatalysts [124]. Depending on the experimental setup, the solid can be set either under a continuous gas flow [122, 123], or under a static atmosphere [119-121]. Both approaches are complementary. Upon photoexcitation, in situ-monitoring the system through timeresolved IR absorption spectroscopy then allows following photogenerated electrons [120], chemical species adsorbed at the surface of the solid and/or the composition of the surrounding gas phase [122,123], as a function of time under various experimental conditions. Analyses may be qualitative or quantitative. They may focus on a single wavenumber $[120,122]$, or deal with spectra in a broad range of wavenumbers $[119,121]$. If the overall performance of the photocatalyst (in terms of reactant conversion and selectivity to products) is evaluated simultaneously (through gas-IR spectroscopy, gas chromatography and/or mass spectrometry), the methodology is qualified as operando, and is often extremely powerful to enlighten reaction mechanisms and to optimize the photocatalyst design [123, 125]. Such operando studies are most often carried out under continuous gas flow, but are possible also under static atmosphere, if the IR cell works as a batch reactor [126]. 


\subsubsection{Under static atmosphere}

\subsubsection{Monitoring photogenerated electrons at a fixed} wavenumber Yamakata et al. used time-resolved IR absorption spectroscopy to study electron- and hole-capture reactions on $\mathrm{TiO}_{2}$ and $\mathrm{Pt} / \mathrm{TiO}_{2}$ photocatalysts exposed to dioxygen, water vapor or methanol vapor (static atmosphere) [120, 127-129], in particular to enlighten the mechanism of the photocatalytic water-splitting reaction $\left(2 \mathrm{H}_{2} \mathrm{O} \rightarrow 2\right.$ $\mathrm{H}_{2}+\mathrm{O}_{2}$ ) on $\mathrm{Pt} / \mathrm{TiO}_{2}$ at $323 \mathrm{~K}$ [120]. Upon irradiation by a $355 \mathrm{~nm}$ UV-pulse, at time $=0$, the $\mathrm{TiO}_{2}$ and $\mathrm{Pt} / \mathrm{TiO}_{2}$ photocatalysts show a transient IR absorption band from 3000 to $1000 \mathrm{~cm}^{-1}$ (with an intensity monotonically increasing with decreasing wavenumber), which is proposed to result from photogenerated electrons trapped in shallow mid-gap states $[120,127]$. Under vacuum, these electrons then recombine with the complementary holes with a multi-exponential rate $[120,127]$. In the presence of reactants such as dioxygen, water vapor or methanol vapor, however, the decay kinetics of the photogenerated electrons are different, which reflects the reactant-induced electron- and/or hole-capture reactions and thereby provides mechanistic insight. Yamakata et al. monitored the latter decay kinetics by tracking the IR absorbance at $2000 \mathrm{~cm}^{-1}$, under increasing reactant pressures [120, 127-129]. To carry out their studies, Yamakata et al. fixed the catalyst powders on $\mathrm{a} \mathrm{CaF}_{2}$ plate, which they placed into a heatable stainless-steel IR vacuum cell. Within the spectrometer, transmitted IR light was dispersed in a grating monochromator. The IR output was then converted to an electric signal in a MCT detector, of which the response determined the time resolution $(50 \mathrm{~ns})$. UV-irradiation of the sample on the plate was achieved thanks to the third harmonic of a $1064 \mathrm{~nm} \mathrm{Nd:YAG} \mathrm{(neodymium-doped}$ yttrium aluminum garnet) laser, thus with a pump pulse of $355 \mathrm{~nm}$. The authors succeeded in detecting transient IR absorbance changes as small as $10^{-6}$ after irradiation by such a UV-pulse by successively accumulating many pulses (typically 300 ) at $0.1-1 \mathrm{~Hz}$ and then averaging the signal [120, 127-129].

\subsubsection{Monitoring photogenerated electrons through step-scan time-resolved IR and correlating their kinetics with the photocatalytic performance Chen et al.} used step-scan time-resolved FTIR spectroscopy on the nanosecond to second time scale to investigate the mechanism of anaerobic methanol photooxidation for hydrogen production, also called photocatalytic reforming of methanol $\left(\mathrm{CH}_{3} \mathrm{OH}+\mathrm{H}_{2} \mathrm{O} \rightarrow \mathrm{CO}_{2}+3 \mathrm{H}_{2}\right)$, on $\mathrm{Pt} / \mathrm{TiO}_{2}$ at room temperature [121, 130]. These authors combined their time-resolved FTIR experiments under methanol and water vapors (static atmosphere), focused on the slow kinetics of long-lived photogenerated electrons, with classical in situ FTIR experiments on the same experimental setup and with photocatalytic tests in methanol-water solution on a different setup, to correlate the decay of the long-lived photogenerated electrons with the steady-state photocatalytic activity of $\mathrm{Pt} / \mathrm{TiO}_{2}$ for hydrogen production [121]. The authors found that electron decays on the millisecond to second time scale correspond to the consumption of these electrons for hydrogen generation, and that molecularly adsorbed methanol or water mediates the proton transfer on the $\mathrm{TiO}_{2}$ surface. To carry out their step-scan time-resolved FTIR experiments, the authors pressed the $\mathrm{TiO}_{2}$ or $\mathrm{Pt} / \mathrm{TiO}_{2}$ samples into selfsupporting wafers, which they placed into a heatable quartz IR cell connected to a vacuum system. The latter allowed evacuating the samples and then introducing methanol and water vapors. The samples were photoexcited by irradiation with the third harmonic of a $1064 \mathrm{~nm} \mathrm{Nd:YAG} \mathrm{pulse} \mathrm{laser,}$ thus at $355 \mathrm{~nm}(3 \mathrm{~Hz}, 8 \mathrm{~mJ} /$ pulse $)$. To prevent the scattered light from reaching interferometer and detector optics, antireflective-coated $\mathrm{Ge}$ plates were placed in the openings of the sample chamber. The synchronization between laser excitation and data acquisition was achieved by means of a pulse generator. As they described in details, the authors adapted the step-scan measurement method to overcome the difficulty of observing also the slow signals ranging from milliseconds to seconds due to the limited low cutoff frequency of the AC amplifier in the MCT detector [121].

\subsubsection{Monitoring adsorbates by rapid-scan and step-scan} time-resolved IR Frei and his team have worked a lot on in situ FTIR spectroscopy upon photoexciting molecular sieves (typically aluminophosphates and zeolites) into which gaseous reactants were loaded, always using the same kind of experimental setup [119, 131-137]. For instance, through combined rapid-scan and step-scan measurements on the microsecond to second time scale, Yeom and Frei probed the mechanism of the ligand-to-metal charge transfer (LMCT) induced reaction of methanol and dioxygen at the gas-micropore interface of a $\mathrm{FeAlPO}_{4}-5$ sieve at $250 \mathrm{~K}$ [119]. Using a heatable IR vacuum cell for analyses under static atmosphere, as Yamakata et al. and Chen et al. did in their above-mentioned works, Yeom and Frei focused on monitoring adsorbates/intermediates/products (e.g., formate $\mathrm{HCO}_{2}^{-}-\mathrm{Fe}$ at $1625 \mathrm{~cm}^{-1}$; formaldehyde $\mathrm{CH}_{2}=\mathrm{O}$ at $1726 \mathrm{~cm}^{-1}$; formic acid $\mathrm{HCO}_{2} \mathrm{H}$ at $1679 \mathrm{~cm}^{-1}$; water $\mathrm{H}_{2} \mathrm{O}$ at $1650 \mathrm{~cm}^{-1}$; hydroxymethyl hydroperoxide $\mathrm{HO}_{2} \mathrm{CH}_{2} \mathrm{OH}$ at $1455 \mathrm{~cm}^{-1}$ ) rather than photogenerated electrons. Their time-resolved study revealed hydroxymethyl hydroperoxide $\left(\mathrm{HO}_{2} \mathrm{CH}_{2} \mathrm{OH}\right)$ to be the main two-electron transfer intermediate. The latter quickly rises on the sub-millisecond time scale, suggesting that it is formed by direct coupling of $\mathrm{HOO}$ and $\mathrm{CH}_{2} \mathrm{OH}$ radicals, the presumed one-electron-transfer products of the interaction of methanol and dioxygen with the excited framework Fe centers. This implies that the photogenerated $\mathrm{Fe}^{+\mathrm{II}}-\mathrm{O}^{-\mathrm{I}}$ moiety reacts with the donor (metha- 
nol) and acceptor (dioxygen) on a microseconds time scale or faster. $\mathrm{H}$ atom transfer of $\mathrm{HOO}$ and $\mathrm{CH}_{2} \mathrm{OH}$ radicals to yield $\mathrm{CH}_{2}=\mathrm{O}$ and $\mathrm{H}_{2} \mathrm{O}_{2}$ appears to be only a minor reaction pathway [119]. In the latter study, Yeom and Frei mounted self-supporting pellets of the $\mathrm{FeAlPO}_{4}-5$ sieve in the abovementioned IR vacuum cell. The IR cell was placed inside an Oxford Cryostat spectrometer allowing for temperature variations between 77 and $473 \mathrm{~K}$. The photoreaction was induced by $355 \mathrm{~nm}$ nanosecond pulses of the third harmonic of a $1064 \mathrm{~nm} \mathrm{Nd:YAG} \mathrm{laser.} \mathrm{A} \mathrm{small} \mathrm{quartz} \mathrm{prism}$ ( $1 \mathrm{~cm}$ edge-to-edge) was used to align the photolysis beam collinearly with the IR probe beam. To prevent photolysis light entering the detector or interferometer compartment, the ports of the latter compartments were closed off by Ge plates with dielectric coatings that maximize IR transmission. The start of the interferogram acquisition following a laser pulse was triggered by an optical pulse diverted from the photolysis laser beam. To ensure that the time delay between the laser pulse and the start of the interferogram acquisition was always the same after each laser pulse, the Nd:YAG laser was triggered by a TTL (transistor-transistor logic) pulse from the FTIR instrument coincident with the forward turn of the moving mirror [119, 138]. Depending on the time resolution, the authors used different procedures to perform their rapid-scan and step-scan measurements (differing by the order of the different steps, their duration, the delay in-between them, etc.), which they described in a very detailed way [119].

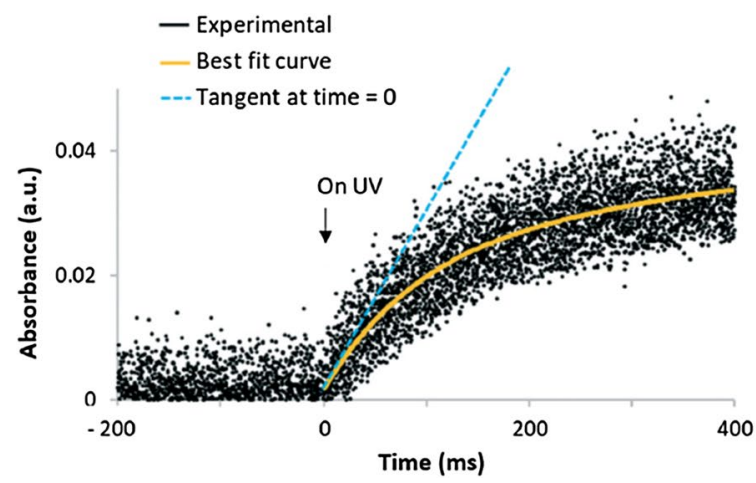

Fig. 9 QCL beam absorbance at $1360 \mathrm{~cm}^{-1}$ vs. time (1 point every $100 \mu \mathrm{s}$ ) over $\mathrm{TiO}_{2}$ P25 during a UV on-off cycle (UV on: left panel, UV off: right panel) under a reaction flow at room temperature and atmospheric pressure. Conditions: flow $=30 \mathrm{~mL} \mathrm{~min}-1 ; 0.1$ and 20 vol\% of methanol and oxygen in argon, respectively; $\mathrm{Hg}-\mathrm{Xe}$ lamp $(200 \mathrm{~W}) ; I_{0} \approx 200 \mathrm{~mW} \mathrm{~cm}{ }^{-2}$. In the left panel, time $=0$ corresponds to the moment when the shutter in-between the lamp and the UVlight guide directed to the IR cell was opened to irradiate the sample. During the preceding $200 \mathrm{~ms}$, the sample was already under a reac-

\subsubsection{Under continuous gas flow}

3.2.2.1 Monitoring adsorbates at a fixed wavenumber and correlating their kinetics with the photocatalytic performance Besides the above-mentioned studies which are all based on the use of IR cells connected to a vacuum system allowing for evacuation of the sample and subsequent loading with the gaseous reactants, there are also systems where time-resolved IR absorption spectroscopy following photoexcitation is performed under continuous gas flow. For instance, Schnee et al. studied the gas-phase photooxidation of methanol over $\mathrm{TiO}_{2}$ photocatalysts at room temperature under flowing methanol and dioxygen (atmospheric pressure), thanks to an appropriate homemade "sandwich" IR reactor-cell positioned within a new quantum cascade lasers (QCLs)-assisted FTIR setup [61, 122, 123]. More precisely, Schnee et al. used a QCL tuned at $1360 \mathrm{~cm}^{-1}$ to monitor the formation and reaction of intermediate surface formate species at a microsecond time resolution over three different $\mathrm{TiO}_{2}$ photocatalysts ( $\mathrm{P} 25$, commercial anatase, and homemade anatase) through UV on-off cycles under flowing methanol and oxygen. Figure 9 shows typical IR absorbance curves vs. time measured for $\mathrm{TiO}_{2} \mathrm{P} 25$ (at 1 point every $100 \mu$ s; the setup being actually able to record 1 point every $5 \mu \mathrm{s})$.

The QCL was actually part of a MIRcat-QT system containing four QCLs emitting in different slightly overlapping ranges, that the authors had coupled with their standard rapid-scan FTIR spectrometer, equipped with a MCT detector. The QCL beam was directed to the sample compartment

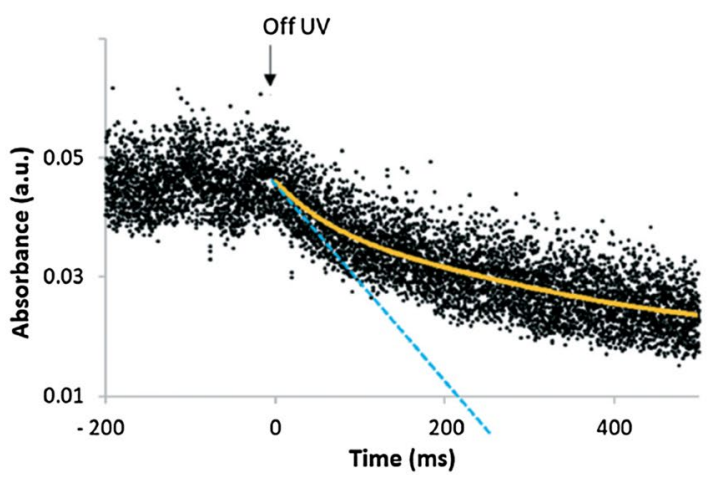

tion flow but still in the dark. In the right panel, time $=0$ corresponds to the moment when the shutter was closed again, still under a reaction flow. The fall times to open and close the shutter were around $6.5 \mathrm{~ms}$ and $3.2 \mathrm{~ms}$, respectively, with a minimum pulse width of $13 \mathrm{~ms}$ [122]. Figure above has been adapted from Fig. 2 contained in Catalysis Science \& Technology 10, 5618-5627 (2020). No permissions request needed as one of the present authors was the author of the reference material, as stated by RSC Licences, copyright \& permissions guidelines 


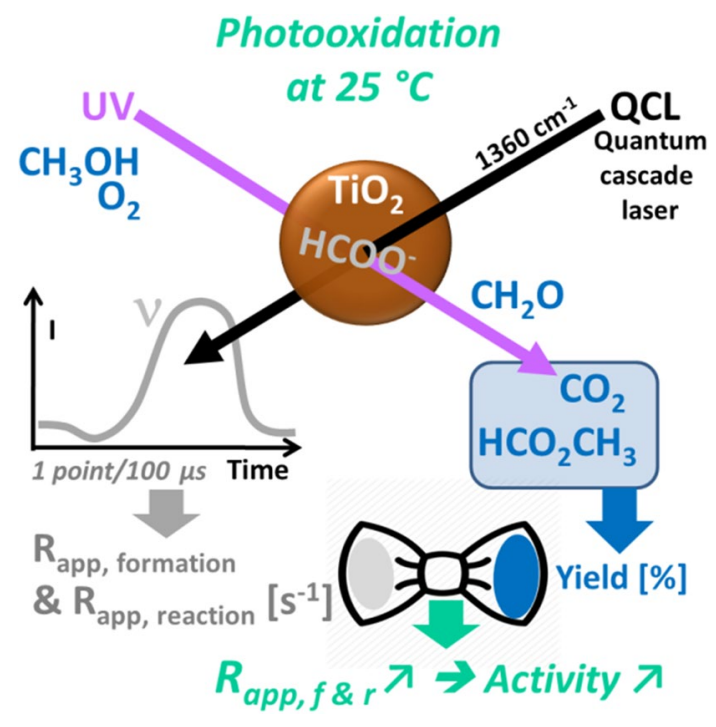

Fig. 10 Schematic summary of the study of Schnee et al. on the gasphase methanol photooxidation at room temperature over $\mathrm{TiO}_{2}$ photocatalysts (pelletized) by means of time-resolved QCL diagnostic at

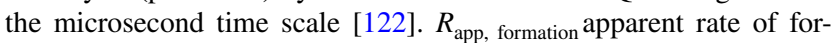
mation, $R_{\text {app, reaction }}$ apparent rate of reaction. Figure above has been adapted from the graphical abstract of Catalysis Science \& Technology 10, 5618-5627 (2020) (https://doi.org/10.1039/D0CY00865F). No permissions request needed as one of the present authors was the author of the reference material, as stated by RSC Licences, copyright \& permissions guidelines

of the spectrometer through the latter but without crossing the interferometer. It was pulsed to avoid local sample heating, and the data acquisition was triggered at each laser pulse. The time resolution thus corresponded to the time in-between two successive laser pulses. The pelletized photocatalysts were irradiated with the polychromatic UV-light of a Xe-Hg lamp, using a UV-light guide mounted at the entrance of the IR reactor-cell and flashing the full surface of the wafer. It was checked that the catalyst was homogeneously illuminated and behaved as a true photo-reactor [122]. Based on the tangent at time $=0$ to the recorded IR absorbance curves vs. time (Fig. 9, for example), the authors were able to determine apparent rates of formation and reaction of the surface formate species, which they finally correlated with the overall steady-state catalytic performance (in terms of total yield of methylformate and carbon dioxide) of the $\mathrm{TiO}_{2}$ photocatalysts measured in subsequent experiments on the same setup under the flowing reactants (as schematically summarized in Fig. 10). Indeed, the reactor-cell was connected to an auxiliary bench of the IR spectrometer equipped with a gas microcell to analyze the composition of the outlet gas flow. The authors found that, the faster the reaction of the surface formates, the higher the catalytic methanol oxidation activity. Thereby, they demonstrated, to their knowledge for the first time, the direct relationship between the conversion of surface formate species and the catalytic performance of the photocatalysts [122]. The above-mentioned "sandwich" transmission IR reactor-cell was equipped with $\mathrm{KBr}$ windows at each side of the sample holder and with a heating system. It was connected to a gas distribution system equipped with mass flow controllers to introduce gases into the lines. The partial pressure of methanol was established using a saturator. In the catalytic performance tests, the pellets were irradiated at $365 \mathrm{~nm}$ thanks to a monochromatic $365 \mathrm{~nm}$ band pass filter to keep the methanol conversion low enough to enable a proper comparison of the different samples [122].

\subsubsection{Operando monitoring the photocatalytic pro-} cess El-Roz et al. investigated the gas-phase photooxidation of methanol, $n$-hexane and carbon monoxide over $\mathrm{TiO}_{2}$-based photocatalysts at room temperature up to $473 \mathrm{~K}$ under continuous gas flow using a FTIR setup similar to that used by Schnee et al. but without being coupled with QCLs [123, 125]. Under photocatalytic reaction conditions, in real time, they simultaneously monitored the species adsorbed on the surface of the photocatalysts by timeresolved FTIR spectroscopy, and the reaction products by gas-phase IR spectroscopy and mass spectrometry, which makes their work truly operando. Time-resolved FTIR spectroscopy was performed at the second to minute time scale. The authors varied parameters such as the reaction temperature, the reactant concentration, the UV-irradiation intensity, and evaluated the effects of these parameters on the photocatalytic reaction [123, 125]. Among their main conclusions brought by the operando approach, they found that the amount of $\mathrm{TiOH}$ surface groups influences the $\mathrm{TiO}_{2}$ band gap, and that these sites (especially the $\mathrm{Ti}^{(\mathrm{IV})} \mathrm{OH}$ ones) improve the chemisorption of methanol (hydrophilic) and favor its photooxidation [125]. Moreover, they found that, for $\mathrm{TiO}_{2}$ photocatalysts, high temperatures (>443 K) favor the chemisorption of methanol and thereby lead to a slight decrease of reactivity. On the contrary, when $\mathrm{TiO}_{2}$ is supported on a BEA zeolite, the reactivity increases with increasing temperature, which was attributed to a decreased poisoning of the $\mathrm{TiO}_{2}$ surface thanks to the BEA support [123].

\subsection{Cis-trans isomerization in molecular photoswitches}

Molecular photoswitches are photochromic compounds that respond reversibly to irradiation with a change of molecular characteristics such as structure, dipole moment, polarity and charge [139]. These light sensitive molecules can be applied in a variety of fields, ranging from the development of light responsive materials, to photocatalysis, and medicine. Among the possible light-induced chemical 
transformations, cis-trans isomerization is an elementary reaction of fundamental importance in triggering the actions of several photoswitches and bio-photoreceptors. Considering the large structural changes and the fast timescale associated to this photoreaction, transient IR spectroscopy appears as a particularly suited technique to investigate its mechanism and dynamics, and has been extensively applied to study photoinduced isomerization both in photoactive proteins, such as GFP [140-144], PYP [145, 146], retinal proteins [12, 147-151], bacteriorhodopsin [152], and in small molecular photoswitches in condensed phase. The literature on the argument is extremely rich, so that we will limit this paragraph to the description of only a few, mostly recent, examples.

One of the first applications of TRIR spectroscopy for studying the photoinduced isomerization of a molecular photoswitch dates back to 1997, when Hamm and co-authors applied this technique to investigate the photochemistry of azobenzene in solution [153]. Although the measurements did not have the necessary time resolution to resolve the photochemical event, the analysis of the spectral evolution of the excited-state IR absorption bands allowed to gain important information concerning the vibrational relaxation of the hot molecules produced upon photoisomerization and to conclude, on the basis of a developed kinetic model, that the system undergoes to a very fast internal vibrational relaxation (IVR), followed by a slower cooling process associated with energy transfer towards the surrounding solvent.

In more recent years, numerous studies appeared where ultrafast TRIR spectroscopy has been used to elucidate the timescale and mechanism of photoinduced isomerization in molecular photoswitches.

Among the recently developed photoswitches, hemithioindigos are certainly gaining increasing importance [154]. These systems, structurally composed by half a stilbene moiety and half a thioindigo unit, undergo a photoinduced isomerization among the central $\mathrm{C}=\mathrm{C}$ bond. The isomerization mechanism of several substituted hemithioindigos has been recently analyzed through ultrafast TRIR spectroscopy. In particular, following the solvent-dependent time evolution of an excited-state absorption band assigned to the carbonyl group present on the structure, it has been possible to evidence the formation of a twisted intra-molecular charge transfer state (TICT) upon photoexcitation [155]. In polar solvents, the formation of TICT can be highlighted from the observed red shift of the $\mathrm{C}=\mathrm{O}$ band, occurring on a timescale of 2-3 ps. Indeed, upon photoexcitation and formation of the charge transfer state, a higher amount of negative charge is localized on the $\mathrm{C}=\mathrm{O}$ moiety: this decreases its bond strength and causes the observed red shift.

Other recent examples concern with the analysis of the reaction mechanism of molecular motors, and in particular of overcrowded alkenes [156]. These systems undergo a combined photoexcited/thermal unidirectional rotational cycle, starting with the photoinduced isomerization around the central $\mathrm{C}=\mathrm{C}$ bond. Comparison of TRIR spectra with FTIR difference spectra of the most stable and metastable isomers allowed to conclude that the metastable isomer forms within $30 \mathrm{ps}$. Furthermore, the observation of a strong broad signal covering the entire investigated spectral region and rapidly decaying within a few picoseconds, suggested the participation of an electronic dark state in the photoinduced reaction. Theoretical computations supported this interpretation, suggesting that the efficiency of the motor is highly influenced by the interaction between optically bright and dark states.

In the continuous search for molecular photoswitches activated with visible or near-IR light, a novel class of molecules, so-called donor-acceptor Stenhouse adducts (DASAs), was recently introduced [157-160]. DASAs are based on a cyclic acceptor (Meldrum's acid or dimethylbarbituric acid) connected to a secondary amine-donor through a triene bridge and have an absorption maximum spanning in the 545-650 $\mathrm{nm}$ range. Upon irradiation, the colored elongated triene form cyclizes to a colorless form. Previous spectroscopic characterization based on UV/Vis and NMR spectroscopy, identified an important transient intermediate in the DASA photoswitching mechanism, proposed to result from a photochemical trans-cis isomerization, whose exact structure could, however, not be resolved [161].
Fig. 11 Possible photoinduced reaction paths in DASA photoswitches. This figure has been adapted with permission from https://doi.org/10.1021/jacs. 7b09081. Further permission related to the material excerpted should be directed to the ACS

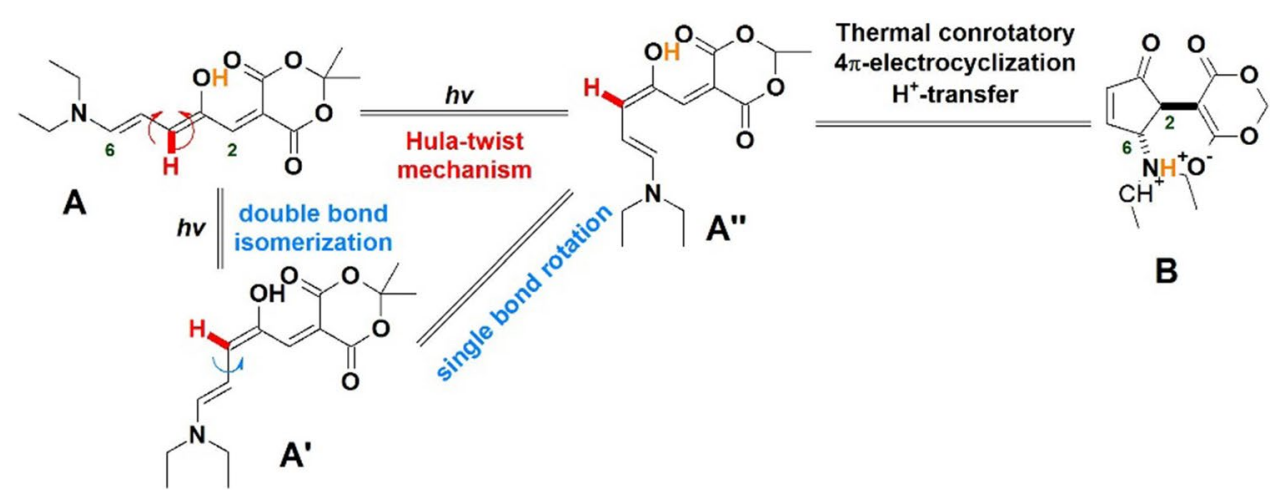



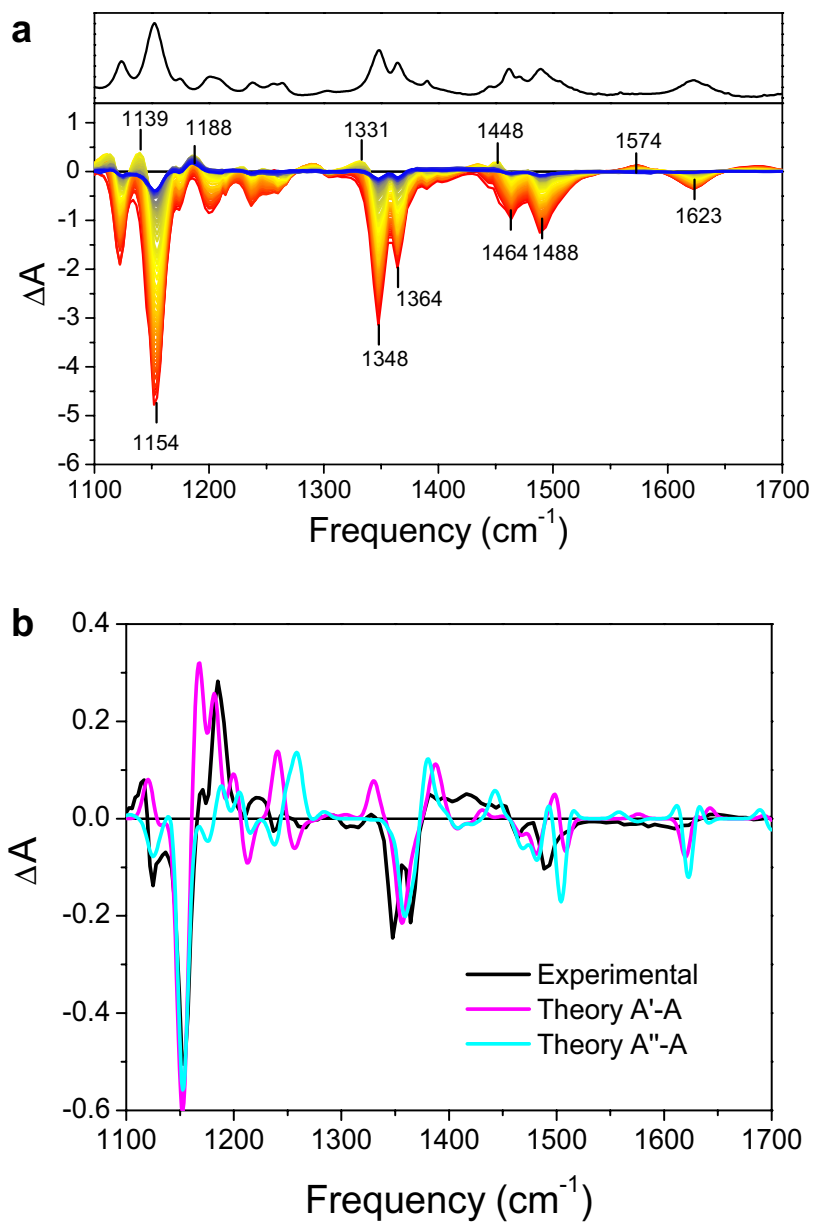

Fig. 12 a ultrafast transient IR spectra measured for dimethyl-Meldrum DASA in chloroform upon excitation at $510 \mathrm{~nm}$; the FTIR spectrum of the A species is reported on top of the transient spectra. Color code from red to blue represents the evolution from 0 up to a 500 ps pump-probe delay. b comparison of the long-living transient IR spectrum of DASA with the computed $\left(\mathrm{A}^{\prime}-\mathrm{A}\right)$ and $\left(\mathrm{A}^{\prime}-\mathrm{A}\right)$ FTIR difference spectra. Panel $\mathbf{b}$ has been adapted with permission from https://doi.org/10.1021/jacs.7b09081. Further permission related to the material excerpted should be directed to the ACS

One of the challenges with DASA is the possibility of isomerization through different $\mathrm{C}=\mathrm{C}$ bonds. Furthermore, reaching the intermediate structure promoting cyclization requires both the isomerization around the central $\mathrm{C}=\mathrm{C}$ bond and the rotation around a single $\mathrm{C}-\mathrm{C}$ bond, as shown in Fig. 11.

The photoinduced reaction could in principle proceed either through a sequential double bond isomerization and single bond rotation forming both the intermediates $\mathrm{A}^{\prime}$ and $\mathrm{A}^{\prime \prime}$ or through a concerted hula-twist mechanism directly forming $\mathrm{A}^{\prime}$.

Ultrafast vis-pump/Mid-IR-probe spectroscopy was employed to clarify the photoswitching mechanism of DASAs. The analysis of the recorded transient spectra, reported in Fig. 12a), evidenced that upon photoexcitation a new product is quickly formed on a 2 ps timescale, as the result of a fast excited-state decay, most probably associated with the reaching of a conical intersection [162]. The system subsequently undergoes vibrational cooling on a timescale of about 10 ps. To identify the nature of the intermediate species formed upon photoexcitation, the long-living transient difference spectrum, which does not decay on the timescale of the experiment ( $1 \mathrm{~ns})$ has been compared with the DFT computed IR difference spectra of the ground-state species A with the two possible $\mathrm{A}^{\prime}$ and $\mathrm{A}^{\prime \prime}$ products (see Fig. 12b). Although the computed spectra of $\mathrm{A}^{\prime}$ and $\mathrm{A}^{\prime \prime}$ present several similarities, the presence of a positive band at $1188 \mathrm{~cm}^{-1}$ in the long-living experimental difference spectrum allowed to unambiguously identify the chemical nature of the intermediate and to establish the dynamics of its formation and subsequent evolution towards the colorless cyclized form, leading to exclude the hula-twist mechanism and confirming that the reaction proceeds through the initial fast formation of the $\mathrm{A}^{\prime}$ isomer (Fig. 12).

Furthermore, by recording transient IR spectra at low temperature $\left(-40^{\circ} \mathrm{C}\right)$ under continuous illumination conditions, it was possible to trap the intermediate $\mathrm{A}^{\prime}$ and selectively excite it to study its own photochemistry, demonstrating the reversibility of the actinic step of DASA's photoswitching reaction. Finally, a subsequent study by the same authors analyzed the thermal steps of the reaction, using rapid-scan FTIR spectroscopy. The analysis of the recorded spectra evidenced a complex reaction path, leading to the formation of different isomers, whose relative stability can be highly influenced by the nature of the solvent [163].

\subsection{Charge carrier and thermal relaxation characterization in dye-sensitized solar cell, optoelectronic/semiconductor and perovskite materials}

\subsubsection{Dye-sensitized solar cells}

Time-resolved IR spectroscopy has revealed as a very powerful tool to get insight into charge transfer processes at the inter- and intra-molecular excited-state level, especially for transition metal complexes in solution [164-168]. The sensitivity offered by transient infrared in the study of charge transfer processes lies in the high specificity of the frequency variation of the vibrations of the ligands due to the electron density shift, for example induced by the absorption of a photon. Within such framework, ultrafast transient IR spectroscopy has been successfully applied, for example, to the characterization of the charge injection processes form excited-state metal complexes to semiconductor nanocrystals for dye-sensitized solar cell (DSSC) applications [169-174]. Asbury et al. [169] were among the first pioneers to use femtosecond IR spectroscopy to study the electron injection 


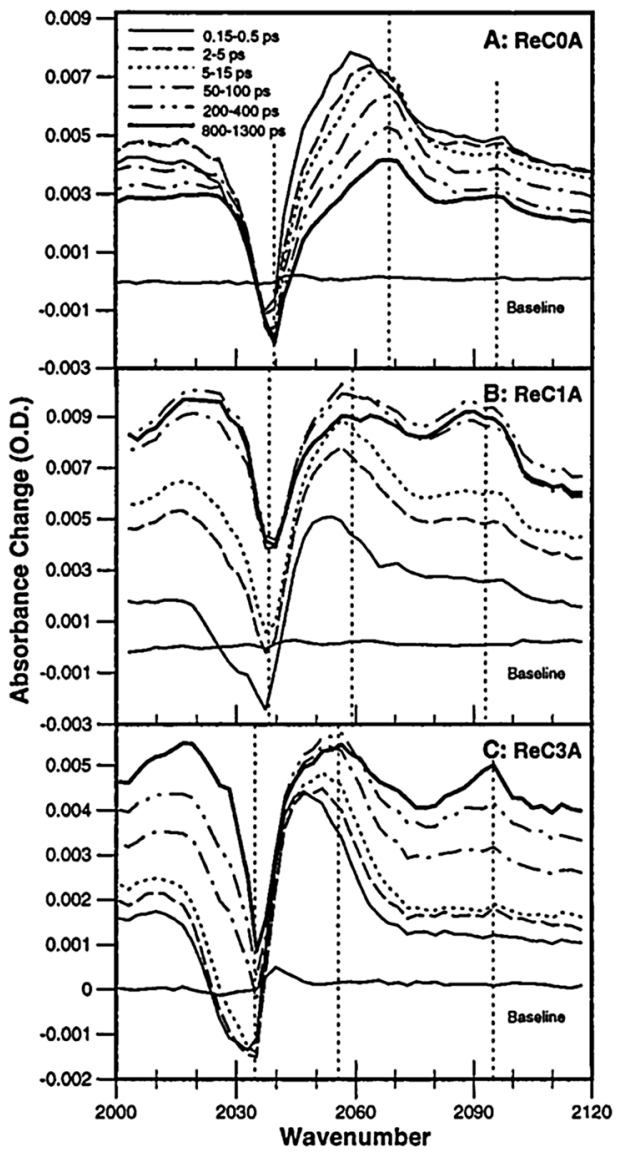

Fig. 13 The (left panel) comparison of transient IR difference spectra of A ReC0A, B ReC1A, and $\mathbf{C ~ R e C 3 A}$ on $\mathrm{TiO}_{2}$ film. The displayed spectra are averages of about 10 times delays in the indicated ranges $0.15-0.5 \mathrm{ps}$ (solid), $2-5 \mathrm{ps}$ (dashed), 5-15 ps (dotted), 50-100 ps (dotted dashed), 200-400 ps (double dotted dashed), and $800-1300$ ps (thick solid). The spectrum at zero delay time is shown as the baseline. The vertical dotted lines indicate the peak positions of the CO stretch bands. (Right panel) Comparison of electron injection

dynamics of $\operatorname{Re}(\mathrm{CO})_{3} \mathrm{Cl}(\mathrm{dcbpy})(\mathrm{ReC} 0 \mathrm{~A})$ and related derivatives on the surface of $\mathrm{TiO}_{2}$ nanocrystalline thin films. It was very well known [175] that free electron were characterized by a broad and unstructured absorption extending in the mid-IR and the authors exploit the rise of such absorption signal to obtain the time scale of electron injection (Fig. 13, right panel). Furthermore, such metal complexes showed one well-isolated CO vibration around $2040 \mathrm{~cm}^{-1}$ in the ground state. After photoexcitation, the charge injected in $\mathrm{TiO}_{2}$ generated an oxidized metal complex where $\mathrm{CO}$ stretching vibrates at higher frequency. Looking at the formation dynamics of excited-state absorption at $2097 \mathrm{~cm}^{-1}$, the authors observed the ligand response to the photoexcitation, obtaining an indirect measurement of the electron injection time. Electron injection from $\mathrm{ReC0A}$ to $\mathrm{TiO}_{2}$ was found to occur on the $<100$-fs time scale, whereas the excited-state vibrational spectrum of the complex evolved

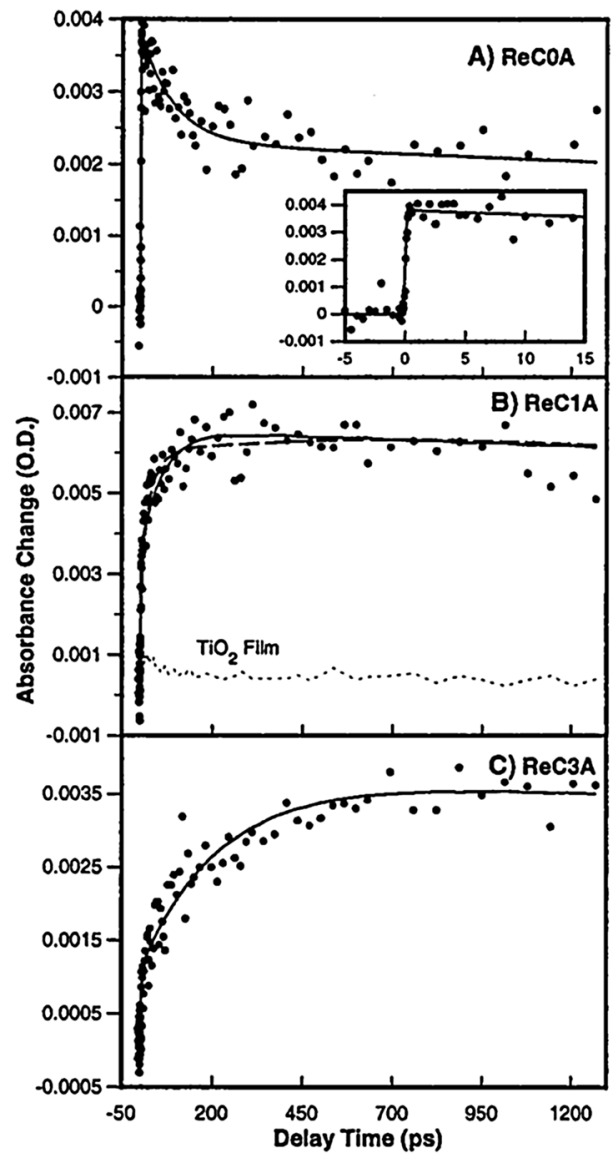

kinetics measured for the injected electron at $2120 \mathrm{~cm}^{-1}$ of A ReC0A, B ReC1A, and C ReC3A on $\mathrm{TiO}_{2}$ film. The dots are experimental data and the solid and dashed curves are fits. The dotted curve in panel $\mathrm{B}$ indicates the signal in unsensitized $\mathrm{TiO}_{2}$ films under the same conditions. The inset in panel A is the same data plotted on a shorter time scale. Figure above has been reprinted (adapted) with permission from The Journal of physical chemistry B 104, 11,957-11,964 (2000). Copyright 2000 American Chemical Society

with a time constant of a few picoseconds, indicating that injection occurred before complete vibrational relaxation in this system. Finally, the injection time was observed to increase as the distance between the metal center and the $\mathrm{TiO}_{2}$ surface is increased by modulating ( $\mathrm{ReC} 0 \mathrm{~A}$ to $\mathrm{ReC} 3 \mathrm{~A}$ ) the length of the aliphatic chain that connect the BPY unit to the $-\mathrm{COOH}$ group that bind the $\mathrm{TiO}_{2}$ surface.

\subsubsection{Organic semiconductors}

The nature of primary photo-excitations in organic semiconductors, particularly in conjugated polymers, has been the subject of an active debate in the scientific community for more than 3 decades [176-179]. The mechanisms underlying photogeneration of charge carriers as well as thermalization promptly after photoexcitation are indicative of the strength of electron-electron interactions relative to the bandwidth 
(as manifested by the exciton binding energy), while carriers transport, trapping, and recombination processes are intimately related to the degree of order, purity, and morphology of the materials. Besides their fundamental interest, these processes determine the overall efficiency of organic electronic devices, such as OLED and organic photovoltaic cells. As the field of organic semiconductors evolved through the initial fundamental studies of molecular crystals to the more application-oriented investigation of conjugated polymers, donor-acceptor and hybrid organic-inorganic systems, ultrafast transient IR techniques have been widely employed toward the experimental characterization of the charge generation and transport properties of new materials and material combinations $[13,180]$. If on one side, the measurement of charge carriers generation and transportation behavior in polydiacetylene revealed itself a good model for subsequent highly ordered organic semiconductors [176], on the other side theoretical description of organic semiconductors was and is still controversial. Indeed, rather than direct generation of free electrons and holes by inter-band photoexcitation as in conventional inorganic semiconductors, the optical excitation in organic semiconductors is generally believed to be excitonic, leading to the formation of spatially localized bound electron-hole pairs (excitons). Within this framework, transient infrared spectroscopy revealed itself a very helpful techniques, capable to monitor the infrared-active vibrational (IRAV) modes, whose intensity and temporal evolution are intimately connected with the charge carrier photogeneration. Indeed, when charge carriers are photogenerated for example on the backbone of a conjugated polymer, they break the local symmetry and thereby transform the even parity Raman active vibrational modes into odd-parity infrared-active modes (IRAV). Consequently, the IRAV modes can be distinguished from the "normal" IR-active and because the IRAV modes are turned on by local charges, the strength of the IRAV absorption provides a probe for the carrier density generated either by chemical doping or photoexcitation [180].

The observation of direct photogeneration of intrinsic delocalized free carriers in small-molecule organic semiconductors has been a long-sought but unsolved issue, of fundamental significance for applications in photo-electric devices and although the excitonic description of photoexcitation in these materials has been widely accepted, this concept is challenged by recently reported phenomena. He et al. [177] reported the observation of direct delocalized free carriers generation upon inter-band photoexcitation in highly crystalline zinc-phthalocyanine films prepared by the weak epitaxy growth method using ultrafast spectroscopy. Transient absorption spectra recorded from the visible to the mid-IR region revealed the existence of short-lived free electrons and holes with a diffusion length estimated to cross at least 11 molecules along the $\pi-\pi$ stacking direction, that subsequently localize to form charge transfer excitons. Their results suggest that delocalized free carriers photogeneration can also be achieved in organic semiconductors when the molecules are packed properly.

\subsubsection{Perovskite thin-film characterization}

Solar cells based on organometal-halide perovskites such as $\mathrm{CH}_{3} \mathrm{NH}_{3} \mathrm{PbI}_{3}$ have emerged as a promising next-generation photovoltaic system, but the underlying photo-physics and photochemistry remain not fully understood due to the limited availability of methods to characterize charge carriers and ions that play a crucial role in the operating device [181-183].

Different from conventional inorganic semiconductors, hybrid organic-inorganic perovskites (HOIP) consist of coexisting organic and inorganic sub-lattices, which present disparate atomic masses and bond strengths. The nanoscopic interpenetration of these disparate components, which lack strong electronic and vibrational coupling, presents fundamental challenges to the understanding of charge and heat dissipation. In comparison to the all-inorganic perovskites which primarily use $\mathrm{Cs}^{+}$as the A-site cation, the simultaneous presence in HOIPs of two different (organic/inorganic) weakly coupled sub-lattices have been shown to confer enhanced photoluminescence (PL) properties and longlived hot carrier [184]. Pulsed visible or UV excitation, followed by an IR probe, proved to be a valuable approach to get insight the time scale related to carrier distribution and recombination as well as thermalization process in HOIPs $[185,186]$.

Near-bandgap transient electronic responses were reported to reveal energy dissipation by hot electrons and holes. However, such a measurement does not directly convey information regarding heating of the atoms, or the sublattice response to photoexcitation. Guo et al. [182] used time-resolved spectroscopy to study the lattice thermalization process as well as the phonon population and equilibration processes in methylammonium lead iodide $\left(\mathrm{MAPbI}_{3}\right)$ by probing the vibrational modes of the organic sub-lattice following above-bandgap $(500 \mathrm{~nm})$ optical excitation in both orthorhombic $(80 \mathrm{~K})$ and tetragonal $(205 \mathrm{~K})$ phase. For the orthorhombic phase of $\mathrm{MAPbI}_{3}, \mathrm{~N}-\mathrm{H}$ asymmetric stretching motions give rise to two strong peaks, with the lower and higher frequency modes denoted as mode-I and mode-II (Fig. 14). Experiments were performed upon a $680 \mathrm{~nm}$ irradiation on thick film of $\mathrm{MAPbI}_{3}$ and probing the $\mathrm{N}-\mathrm{H}$ stretching vibrations, that exhibit strong photoinduced changes in dipole moment. The strategy adopted to follow the thermal equilibration process was to induce a relevant local-temperature increase employing a pump fluence capable to generate a high $\left(4.5 \times 10^{18} \mathrm{~cm}^{-3}\right.$ to $93 \times 10^{18}$ $\mathrm{cm}^{-3}$ ) excitation carrier density. Carrier density represents 

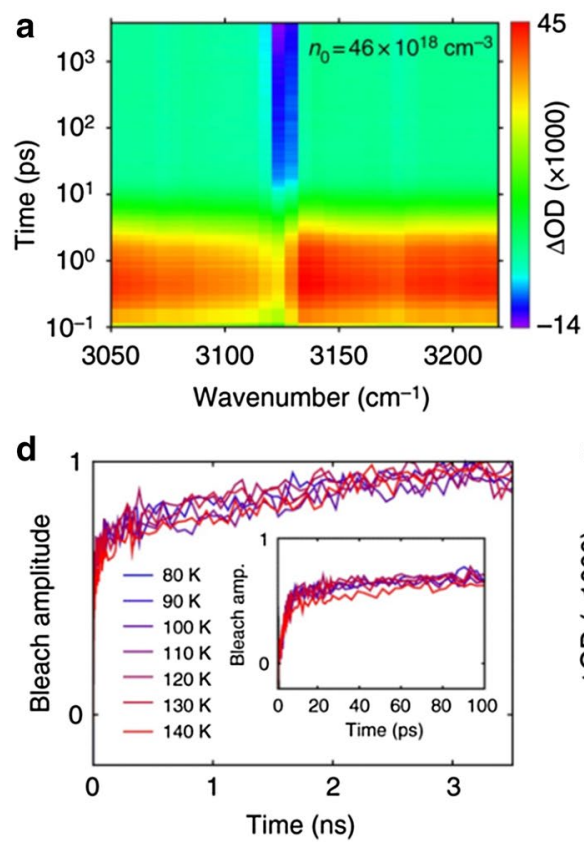
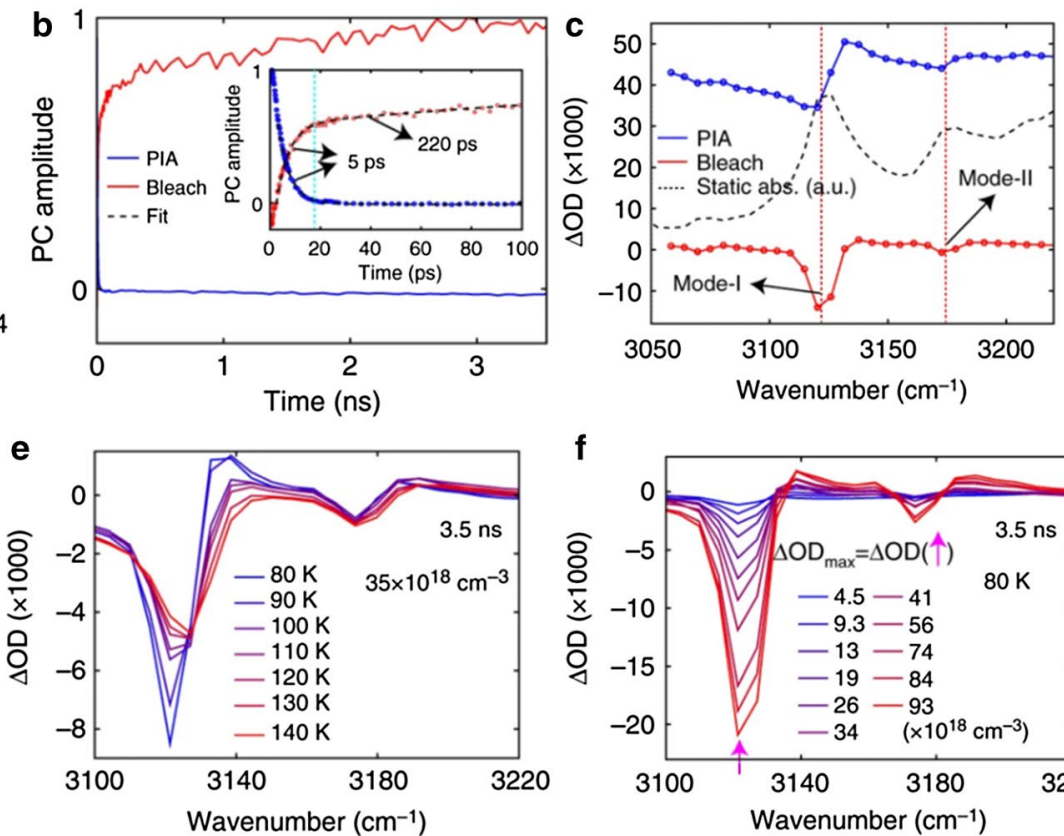

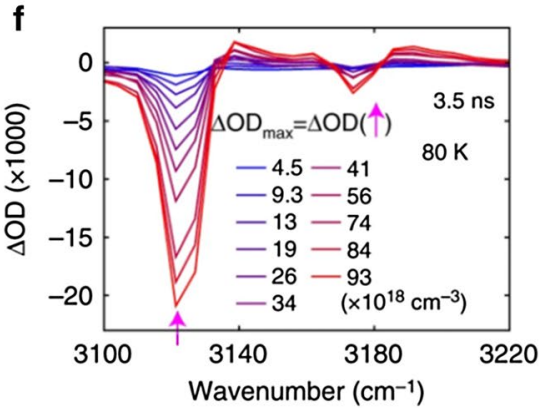

Fig. 14 Transient absorption measurements of MAPbI3. a Transient spectral map of $\Delta \mathrm{OD}$ measured at $80 \mathrm{~K}$ using 500-nm pump excitation with $329 \mu \mathrm{J} \mathrm{cm}{ }^{-2}$ fluence (corresponding to $n_{0}$ of $46 \times 10^{18}$ $\mathrm{cm}^{-3}$ ). b Kinetics of the two PCs up to $3.5 \mathrm{~ns}$. Inset shows the kinetics from 0 to 100 ps. Kinetics of the PIA and bleaching components were fitted with one and two exponentials, respectively (shown as the black-dashed lines in the inset). c Two principle components (PCs) of the transient response shown in red (bleaching) and blue (photoinduced absorption, or PIA). Circles on the blue and red lines correspond to the pixels of the array detector. The black-dashed line shows the absorbance measured with the array detector without pump excitation. d Kinetics of the bleaching component measured at different temperatures using $500 \mathrm{~nm}$ pump under a fixed excitation carrier density of $35 \times 10^{18} \mathrm{~cm}^{-3}$. e Transient spectra at delay time of $3.5 \mathrm{~ns}$ measured at different temperatures under fixed $n_{0}$ of $35 \times 10 \mathrm{~cm}^{-3}$. f Transient spectra at delay time of $3.5 \mathrm{~ns}$ measured at $80 \mathrm{~K}$ under various n0. Figure above has been adapted with permission from Figs. 2 and 4 contained in Nature Communications 9, 2792 (2018), under the Creative Commons Attribution 4.0 International License: http://creat ivecommons.org/licenses/by/4.0/ the number of carriers, per unit of volume, generated right after photoexcitation. It is commonly indicated with $n_{0}$ and was estimated as $n_{0}=\frac{F(1-R)(1-1 / e)}{\hbar \omega l}$ where $l$ is the penetration depth of the pump (taken to be $1 / \alpha$ ), $F$ is the pump fluence, $\hbar \omega$ is the photon energy $(2.48 \mathrm{eV})$ of the pump, and $e$ is the natural logarithm constant; $\alpha$ is the absorption coefficient that, at $500 \mathrm{~nm}$, is close to $1.11 \times 10^{5} \mathrm{~cm}^{-1}$.

Transient spectral response (Fig. 14a, c) was dominated by a short-lived ( $20 \mathrm{ps})$ photoinduced absorption and by a fast (20 ps) and slow (nanoseconds) growing bleaching feature. The short-lived induced absorption has been interpreted as a signature of intra-conduction-band and intra-valenceband absorption by photoexcited carriers. The fast timescale of the bleaching component was attributed to the excitation of low-energy phonon modes (denoted as LEPMs), including various optical phonon modes and acoustic phonon modes, due to hot carrier relaxation. Population of these LEPMs induces thermalization of the hydrogen bond network, which weakens the hydrogen bonds and with it the oscillator strength of the $\mathrm{N}-\mathrm{H}$ vibrational modes. Finally, the slow rise of the bleaching component was attributed to the slow buildup of high-energy phonon modes (denoted as HEPMs) specific to the organic sub-lattice through phonon-phonon interactions between the LEPMs and HEPMs.

Determining whether the dynamically disordered organic cations with large dipole moment influence the optoelectronic properties of $\mathrm{CH}_{3} \mathrm{NH}_{3} \mathrm{PbI}_{3}$ has been an outstanding challenge. Guo et al. [187] used an ultrafast IR pump/visible probe experiment to evaluate the coupling between the organic and inorganic sub-lattice.

\subsection{T-2DIR of metal-carbonyl complexes}

T-2DIR spectroscopy has been used for different samples, one of the most instructive applications being the measurement of T-2DIR spectra of metal-carbonyl complexes. In the following example, Bredenbeck et al. [39] measured the transient 2DIR spectra of a $\left[\operatorname{Re}(\mathrm{CO})_{3}(\mathrm{dmbpy})\right.$ Cl] (dmbpy $=4,4$ '-dimethyl-2,2' bipyridine), which undergoes metal-to-ligand charge transfer (MLCT) upon photoexcitation.

The molecule, sketched in Fig. 15a, has three CO ligands, with three vibrational modes, marked as a'(2), a"' and a'(1) in the FTIR spectrum reported in Fig. 15b. Figure 15c 
Fig. 15 a $\left[\operatorname{Re}(\mathrm{CO})^{-} 3(\mathrm{dmbi}) \mathrm{Cl}\right]$ structure; $\mathbf{b}$ FTIR spectrum of $\left[\operatorname{Re}(\mathrm{CO})^{-} 3(\mathrm{dmbi}) \mathrm{Cl}\right]$, carbonyl region; c 2DIR spectrum of the electronic ground state, $t_{1}=1.5 \mathrm{ps} ; \mathbf{d}$ TRIR spectrum measured 20 ps after excitation at $390 \mathrm{~nm} ; \mathbf{e}$ T-2DIR spectrum measured $20 \mathrm{ps}$ after visible excitation, and IR delay $1.5 \mathrm{ps}$. This figure has been reproduced with permission from https:// doi.org/10.1002/cphc. 20070 0148.39 Copyright (C) 2007 WILEY-VCH Verlag GmbH \& Co. KGaA, Weinheim
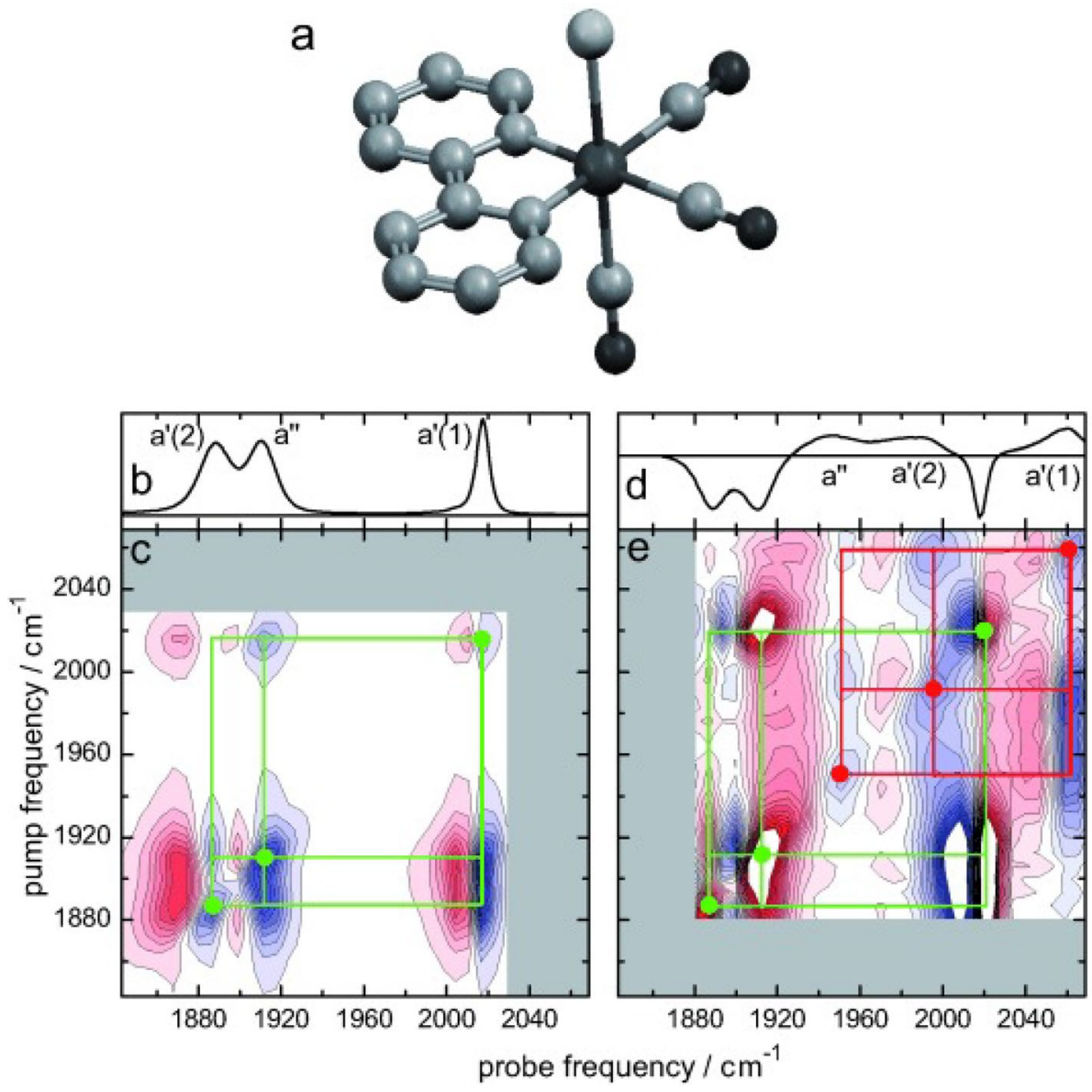

reports the ground-state 2DIR spectrum recorded at a pump-probe delay of $1.5 \mathrm{ps}$. The three $\mathrm{CO}$ modes give rise to three diagonal peaks, marked with green dots. Each diagonal peak features a ground-state bleaching (negative band, blue) and a red-shifted excited-state absorption band (positive band, red). Cross peaks due to coupling among the $\mathrm{CO}$ vibrations are visible out of diagonal, evidenced by the two green squares. Upon visible excitation, the system undergoes a MLCT transition, causing shifts in the carbonyl frequencies. The TRIR spectrum reported in Fig. 15e indeed presents three negative bleaching bands, whose frequencies correspond to those observed in the FTIR spectrum, and new positive excited absorption bands, observed because the charge transfer from the metal core to the bipyridyl ligand induces a blue shift of the $\mathrm{CO}$ absorption frequencies. Figure $15 \mathrm{e}$ reports the T-2DIR spectrum of the system recorded 20 ps after visible excitation. Along the diagonal the same peaks observed in the ground-state 2DIR spectrum are visible, but with opposite sign, being this a double difference spectrum (green dots in Fig. 15e). Furthermore, peaks due to the MLCT state appear along the diagonal, marked with red dots. Couplings among the excited-state signals can be highlighted out of diagonal, as indicated by the red square. This example demonstrates that the complete 2D spectrum of a transient species can be obtained, opening the possibility of monitoring couplings, anharmonicity and transient polarization changes in the excited state.

\section{Conclusions and perspectives}

Time-resolved IR spectroscopy has become nowadays a classical technique for the study of photoinduced reactions. In some specific fields, like photobiology, it is now considered as a nearly mandatory technique, and several research groups presently work on strategies to make non-photoinduced biochemical reactions triggerable by light $[188,189]$. However, the situation is rapidly evolving also in other fields of photochemistry and we believe that soon time-resolved IR will be used by an increasing number of research groups. The possibility of following the reaction mechanism by specific IR bands, united with the possibility of triggering the reaction by laser pulses or other light sources is a definite advantage.

Several recently developed approaches have been described in the methods section, but it should be mentioned that even more revolutionary time-resolved IR techniques are being developed [190, 191]. We believe that in the future the integration of different approaches, covering different 
timescales, or investigating the process under different conditions like flash(es) vs. continuous illumination, will be the key issue.

Signal processing strategies, data treatment, comparison with theoretical calculations are already widely used. In the near future, integration with data coming from other techniques, notably vibrational spectroscopies such as Raman or Resonance Raman, or UV-visible, especially in timeresolved mode, will become more and more common.

\section{Declarations}

Conflict of interest On behalf of all the authors, the corresponding author (A.M.) states that there is no conflict of interest.

\section{References}

1. Goldenstein, C. S., Spearrin, R. M., Jeffries, J. B., \& Hanson, R. K. (2017). Infrared laser-absorption sensing for combustion gases. Progress in Energy and Combustion Science, 60, 132-176.

2. Innocenzi, P., Kidchob, T., Malfatti, L., Costacurta, S., Takahashi, M., Piccinini, M., \& Marcelli, A. (2008). In-situ study of sol-gel processing by time-resolved infrared spectroscopy. Journal of Sol-Gel Science and Technology, 48(1), 253-259.

3. Andanson, J.-M., \& Baiker, A. (2010). Exploring catalytic solid/liquid interfaces by in situ attenuated total reflection infrared spectroscopy. Chemical Society Reviews, 39(12), $4571-4584$

4. Lamberti, C., Groppo, E., Spoto, G., Bordiga, S., \& Zecchina, A. (2007). Infrared spectroscopy of transient surface species. In B. C. Gates \& H. Knözinger (Eds.), Advances in catalysis (Vol. 51, pp. 1-74). Berlin: Academic Press.

5. Avouris, P., Bethune, D. S., Lankard, J. R., Sorokin, P. P., \& Schell-Sórokin, A. J. (1981). Time-resolved IR spectral photography. Journal of Photochemistry, 17(2), 227-232.

6. Hancock, G.; Heard, D. E. (1993). Time-resolved FTIR emission studies of photochemical reactions. In: Advances in photochemistry, pp. 1-65.

7. Hermann, H., Grevels, F. W., Henne, A., \& Schaffner, K. (1982). Flash photolysis with infrared detection. The photochemistry and secondary thermal reactions of $\mathrm{M}(\mathrm{CO}) 6[\mathrm{M}=$ $\mathrm{Cr}, \mathrm{Mo}$, and W]. The Journal of Physical Chemistry, 86(26), 5151-5154.

8. Iwata, K., Kato, C., \& Hamaguchi, H.-O. (1989). Microsecond Time-Resolved Infrared Spectroscopy of a Thin Film of $\tau$-Form Metal-Free Phthalocyanine Fine Particles. Applied Spectroscopy, 43(1), 16-19.

9. Butler, J. M., George, M. W., Schoonover, J. R., Dattelbaum, D. M., \& Meyer, T. J. (2007). Application of transient infrared and near infrared spectroscopy to transition metal complex excited states and intermediates. Coordination Chemistry Reviews, 251(3-4), 492-514.

10. Siebert, F., \& Mäntele, W. (1980). Investigations of the rhodopsin/Meta I and rhodopsin/Meta II transitions of bovine rod outer segments by means of kinetic infrared spectroscopy. Biophysics of Structure and Mechanism, 6(2), 147-164.

11. Weitz, E. (1994). Transient infrared spectroscopy as a probe of coordinatively unsaturated metal carbonyls in the gas phase. The Journal of Physical Chemistry, 98(44), 11256-11264.
12. Nibbering, E. T. J., Fidder, H., \& Pines, E. (2005). ULTRAFAST CHEMISTRY: using time-resolved vibrational spectroscopy for interrogation of structural dynamics. Annual Review of Physical Chemistry, 56(1), 337-367.

13. Munson, K. T., Kennehan, E. R., \& Asbury, J. B. (2019). Structural origins of the electronic properties of materials via timeresolved infrared spectroscopy. Journal of Materials Chemistry C, 7(20), 5889-5909.

14. Groot, M. L., van Wilderen, L. J., \& Di Donato, M. (2007). Timeresolved methods in biophysics. 5. Femtosecond time-resolved and dispersed infrared spectroscopy on proteins. Photochemical \& Photobiological Sciences, 6(5), 501-507.

15. Lorenz-Fonfria, V. A. (2020). Infrared difference spectroscopy of proteins: from bands to bonds. Chemical Reviews, 120(7), $3466-3576$

16. Mezzetti, A. (2020). Photobiological systems studied by timeresolved infrared spectroscopy (2015-2018). In A. Albini \& S. Protti (Eds.), Photochemistry (Vol. 47, pp. 159-195). The Royal Society of Chemistry.

17. Elsaesser, T., \& Kaiser, W. (1986). Visible and infrared spectroscopy of intramolecular proton transfer using picosecond laser pulses. Chemical Physics Letters, 128(3), 231-237.

18. Maiti, S., Cowen, B. R., Diller, R., Iannone, M., Moser, C. C. Dutton, P. L., \& Hochstrasser, R. M. (1993). Picosecond infrared studies of the dynamics of the photosynthetic reaction center. Proceedings of the National Academy of Sciences, 90(11), 5247-5251.

19. Di Donato, M., \& Groot, M. L. (2015). Ultrafast infrared spectroscopy in photosynthesis. Biochimica et Biophysica Acta-Bioenergetics, 1847(1), 2-11.

20. Kaindl, R. A., Wurm, M., Reimann, K., Hamm, P., Weiner, A. M., \& Woerner, M. (2000). Generation, shaping, and characterization of intense femtosecond pulses tunable from 3 to $20 \mu \mathrm{m}$. Journal of the Optical Society of America B, 17(12), 2086-2094.

21. Cerullo, G., \& De Silvestri, S. (2003). Ultrafast optical parametric amplifiers. Review of Scientific Instruments, 74(1), 1-18.

22. Lochbrunner, S.; Wilhelm, T.; Piel, J.; Sporlein, S.; Riedle, E. (1999). In: Sub-20-fs tunable pulses in the visible and NIR by noncollinear optical parametric amplification (NOPA). Advanced Solid State Lasers, Boston, Massachusetts, Optical Society of America: Boston, Massachusetts, p TuA4.

23. Cerullo, G., Nisoli, M., \& De Silvestri, S. (1997). Generation of $11 \mathrm{fs}$ pulses tunable across the visible by optical parametric amplification. Applied Physics Letters, 71(25), 3616-3618.

24. Lim, M., \& Anfinrud, P. A. (2005). Ultrafast time-resolved IR studies of protein-ligand interactions. Methods in Molecular Biology, 305, 243-260.

25. Lim, M., Jackson, T. A., \& Anfinrud, P. A. (2004). Orientational distribution of $\mathrm{CO}$ before and after photolysis of $\mathrm{MbCO}$ and HbCO: A determination using time-resolved polarized MidIR spectroscopy. Journal of the American Chemical Society, 126(25), 7946-7957.

26. Zhang, W.-K. (2016). Polarization dependent time-resolved infrared spectroscopy and its applications. Chinese Journal of Chemical Physics, 29(1), 1-9.

27. Treuffet, J., Kubarych, K. J., Lambry, J.-C., Pilet, E., Masson, J.-B., Martin, J.-L., Vos, M. H., Joffre, M., \& Alexandrou, A. (2007). Direct observation of ligand transfer and bond formation in cytochrome $\mathrm{c}$ oxidase by using mid-infrared chirped-pulse upconversion. Proceedings of the National Academy of Sciences, 104(40), 15705-15710.

28. Baiz, C. R., \& Kubarych, K. J. (2011). Ultrabroadband detection of a mid-IR continuum by chirped-pulse upconversion. Optics Letters, 36(2), 187-189.

29. Zhu, J., Mathes, T., Stahl, A. D., Kennis, J. T. M., \& Groot, M. L. (2012). Ultrafast mid-infrared spectroscopy by chirped pulse 
upconversion in 1800-1000cm-1 region. Optics Express, 20(10), 10562-10571.

30. Anna, J. M., Nee, M. J., Baiz, C. R., McCanne, R., \& Kubarych, K. J. (2010). Measuring absorptive two-dimensional infrared spectra using chirped-pulse upconversion detection. Journal of the Optical Society of America B, 27(3), 382-393.

31. Hamm, P., \& Zanni, M. (2011). Concepts and methods of $2 D$ infrared spectroscopy. Cambridge University Press.

32. Lima, M., Candelaresi, M., \& Foggi, P. (2013). 2D-IR spectroscopy: An additional dimension to investigate ultrafast structural dynamics. Journal of Raman Spectroscopy, 44(10), 1470-1477.

33. Park, S., Kwak, K., \& Fayer, M. D. (2007). Ultrafast 2D-IR vibrational echo spectroscopy: A probe of molecular dynamics. Laser Physics Letters, 4(10), 704-718.

34. Zheng, J., Kwak, K., \& Fayer, M. D. (2007). Ultrafast 2D IR vibrational echo spectroscopy. Accounts of Chemical Research, 40(1), 75-83.

35. Hamm, P., Lim, M., \& Hochstrasser, R. M. (1998). Structure of the amide I band of peptides measured by femtosecond nonlinear-infrared spectroscopy. The Journal of Physical Chemistry B, 102(31), 6123-6138.

36. Lapini, A., Pagliai, M., Fanetti, S., Citroni, M., Scandolo, S., Bini, R., \& Righini, R. (2016). Pressure dependence of hydrogen-bond dynamics in liquid water probed by ultrafast infrared spectroscopy. The Journal of Physical Chemistry Letters, 7(18), 3579-3584.

37. Delor, M., Sazanovich, I. V., Towrie, M., \& Weinstein, J. A. (2015). Probing and exploiting the interplay between nuclear and electronic motion in charge transfer processes. Accounts of Chemical Research, 48(4), 1131-1139.

38. Hunt, N. T. (2014). Transient 2D-IR spectroscopy of inorganic excited states. Dalton Transactions, 43(47), 17578-17589.

39. Bredenbeck, J., Helbing, J., Kolano, C., \& Hamm, P. (2007). Ultrafast 2DIR spectroscopy of transient species. ChemPhysChem, 8(12), 1747-1756.

40. Helbing, J., \& Hamm, P. (2011). Compact implementation of Fourier transform two-dimensional IR spectroscopy without phase ambiguity. Journal of the Optical Society of America B, 28(1), 171-178.

41. DeFlores, L. P., Nicodemus, R. A., \& Tokmakoff, A. (2007). Two-dimensional Fourier transform spectroscopy in the pumpprobe geometry. Optics Letters, 32(20), 2966-2968.

42. Shim, S.-H., \& Zanni, M. T. (2009). How to turn your pumpprobe instrument into a multidimensional spectrometer: 2D IR and Vis spectroscopies via pulse shaping. Physical Chemistry Chemical Physics, 11(5), 748-761.

43. Farrell, K. M., Ostrander, J. S., Jones, A. C., Yakami, B. R., Dicke, S. S., Middleton, C. T., Hamm, P., \& Zanni, M. T. (2020). Shot-to-shot 2D IR spectroscopy at $100 \mathrm{kHz}$ using a $\mathrm{Yb}$ laser and custom-designed electronics. Optics Express, 28(22), 33584-33602.

44. Kumar, S. K. K.; Tamimi, A.; Fayer, M. D., Comparisons of 2D IR measured spectral diffusion in rotating frames using pulse shaping and in the stationary frame using the standard method. The Journal of Chemical Physics 2012, 137 (18), 184201.

45. Shim, S.-H., Strasfeld, D. B., Ling, Y. L., \& Zanni, M. T. (2007). Automated 2D IR spectroscopy using a mid-IR pulse shaper and application of this technology to the human islet amyloid polypeptide. Proceedings of the National Academy of Sciences, 104(36), 14197-14202.

46. Mezzetti, A., Nabedryk, E., Breton, J., Okamura, M. Y., Paddock, M. L., Giacometti, G., \& Leibl, W. (2002). Rapid-scan Fourier transform infrared spectroscopy shows coupling of GLuL212 protonation and electron transfer to QB in Rhodobacter sphaeroides reaction centers. Biochimica et Biophysica Acta (BBA)-Bioenergetics, 1553(3), 320-330.
47. Mezzetti, A., Leibl, W., Breton, J., \& Nabedryk, E. (2003). Photoreduction of the quinone pool in the bacterial photosynthetic membrane: Identification of infrared marker bands for quinol formation. FEBS Letters, 537(1-3), 161-165.

48. Mezzetti, A., Alexandre, M., Thurotte, A., Wilson, A., Gwizdala, M., \& Kirilovsky, D. (2019). Two-step structural changes in orange carotenoid protein photoactivation revealed by timeresolved fourier transform infrared spectroscopy. The Journal of Physical Chemistry B, 123(15), 3259-3266.

49. Uhmann, W., Becker, A., Taran, C., \& Siebert, F. (1991). Timeresolved FT-IR absorption spectroscopy using a step-scan interferometer. Applied Spectroscopy, 45(3), 390-397.

50. Rödig, C., \& Siebert, F. (1999). Errors and artifacts in timeresolved step-scan FT-IR spectroscopy. Applied Spectroscopy, 53(8), 893-901.

51. Mezzetti, A., \& Spezia, R. (2008). Time-resolved step scan FTIR spectroscopy and DFT investigation on triplet formation in peridinin-chlorophyll-a-protein from Amphidinium carterae at low temperature. Spectroscopy, 22(4), 235-250.

52. Griffiths, P. R., Hirsche, B. L., \& Manning, C. J. (1999). Ultrarapid-scanning Fourier transform infrared spectrometry. Vibrational Spectroscopy, 19(1), 165-176.

53. Süss, B., Ringleb, F., \& Heberle, J. (2016). New ultrarapid-scanning interferometer for FT-IR spectroscopy with microsecond time-resolution. Review of Scientific Instruments, 87(6), 063113.

54. Souvignier, G., \& Gerwert, K. (1992). Proton uptake mechanism of bacteriorhodopsin as determined by time-resolved stroboscopic-FTIR-spectroscopy. Biophysical Journal, 63(5), 1393-1405.

55. Noguchi, T., Suzuki, H., Tsuno, M., Sugiura, M., \& Kato, C. (2012). Time-resolved infrared detection of the proton and protein dynamics during photosynthetic oxygen evolution. Biochemistry, 51(15), 3205-3214.

56. Kubo, M., Nakashima, S., Yamaguchi, S., Ogura, T., Mochizuki, M., Kang, J., Tateno, M., Shinzawa-Itoh, K., Kato, K., \& Yoshikawa, S. (2013). Effective pumping proton collection facilitated by a copper site $\left(\mathrm{Cu}_{\mathrm{B}}\right)$ of bovine heart cytochrome $c$ oxidase, revealed by a newly developed time-resolved infrared system. Journal of Biological Chemistry, 288(42), 30259-30269.

57. Rimshaw, A., Grieco, C., \& Asbury, J. B. (2016). High sensitivity nanosecond mid-infrared transient absorption spectrometer enabling low excitation density measurements of electronic materials. Applied Spectroscopy, 70(10), 1726-1732.

58. Dyer, R. B., Einarsdottir, O., Killough, P. M., Lopez-Garriga, J. J., \& Woodruff, W. H. (1989). Transient binding of photodissociated carbon monoxide to $\mathrm{CuB}+$ of eukaryotic cytochrome oxidase at ambient temperature. Direct evidence from time-resolved infrared spectroscopy. Journal of the American Chemical Society, 111(19), 7657-7659.

59. Waried, H. H. (2018). Synchronization of quantum cascade lasers with mutual optoelectronic coupling. Chinese Journal of Physics, 56(3), 1113-1120.

60. Tittel, F. K., Bakhirkin, Y., Kosterev, A. A., \& Wysocki, G. (2006). Recent advances in trace gas detection using quantum and interband cascade lasers. The Review of Laser Engineering, 34(4), 275-282.

61. Schnee, J., Bazin, P., Barviau, B., Grisch, F., Beccard, B. J., \& Daturi, M. (2019). Coupling a rapid-scan FT-IR spectrometer with quantum cascade lasers within a single setup: An easy way to reach microsecond time resolution without losing spectral information. Analytical chemistry, 91(7), 4368-4373.

62. Panman, M. R., Bodis, P., Shaw, D. J., Bakker, B. H., Newton, A. C., Kay, E. R., Leigh, D. A., Buma, W. J., Brouwer, A. M., \& Woutersen, S. (2012). Time-resolved vibrational spectroscopy of a molecular shuttle. Physical Chemistry Chemical Physics, 14(6), 1865-1875. 
63. Chung, H. S., Khalil, M., Smith, A. W., \& Tokmakoff, A. (2007). Transient two-dimensional IR spectrometer for probing nanosecond temperature-jump kinetics. Review of Scientific Instruments, 78(6), 063101.

64. Song, Y., Konar, A., Sechrist, R., Roy, V. P., Duan, R., Dziurgot, J., Policht, V., Matutes, Y. A., Kubarych, K. J., \& Ogilvie, J. P. (2019). Multispectral multidimensional spectrometer spanning the ultraviolet to the mid-infrared. Review of Scientific Instruments, 90(1), 013108.

65. Klocke, J. L., Mangold, M., Allmendinger, P., Hugi, A., Geiser, M., Jouy, P., Faist, J., \& Kottke, T. (2018). Single-shot submicrosecond mid-infrared spectroscopy on protein reactions with quantum cascade laser frequency combs. Analytical Chemistry, 90(17), 10494-10500.

66. Ritter, E., Puskar, L., Kim, S. Y., Park, J. H., Hofmann, K. P., Bartl, F., Hegemann, P., \& Schade, U. (2019). Féry infrared spectrometer for single-shot analysis of protein dynamics. The Journal of Physical Chemistry Letters, 10(24), 7672-7677.

67. Lesage, T., Verrier, C., Bazin, P., Saussey, J., \& Daturi, M. (2003). Studying the NO-trap mechanism over a Pt-Rh/Ba/Al2O3 catalyst by operando FT-IR spectroscopy. Physical Chemistry Chemical Physics, 5(20), 4435-4440.

68. van Stokkum, I. H. M., Larsen, D. S., \& van Grondelle, R. (2004). Global and target analysis of time-resolved spectra. Biochimica et Biophysica Acta (BBA)-Bioenergetics, 1657(2), 82-104.

69. Blanchet, L., Mezzetti, A., Ruckebusch, C., Huvenne, J. P., \& de Juan, A. (2007). Multivariate curve resolution of rapid-scan FTIR difference spectra of quinone photoreduction in bacterial photosynthetic membranes. Analytical and Bioanalytical Chemistry, 387(5), 1863-1873.

70. Blanchet, L., Ruckebusch, C., Mezzetti, A., Huvenne, J. P., \& de Juan, A. (2009). Monitoring and interpretation of photoinduced biochemical processes by rapid-scan FTIR difference spectroscopy and hybrid hard and soft modeling. The Journal of Physical Chemistry B, 113(17), 6031-6040.

71. Lórenz-Fonfría, V. A., \& Kandori, H. (2007). Bayesian maximum entropy (Two-Dimensional) lifetime distribution reconstruction from time-resolved spectroscopic data. Applied Spectroscopy, 61(4), 428-443.

72. Mezzetti, A., Blanchet, L., de Juan, A., Leibl, W., \& Ruckebusch, C. (2011). Ubiquinol formation in isolated photosynthetic reaction centres monitored by time-resolved differential FTIR in combination with 2D correlation spectroscopy and multivariate curve resolution. Analytical and Bioanalytical Chemistry, 399(6), 1999-2014.

73. Mezzetti, A., Kish, E., Robert, B., \& Spezia, R. (2015). Assignment of IR bands of isolated and protein-bound Peridinin in its fundamental and triplet state by static FTIR, time-resolved stepscan FTIR and DFT calculations. Journal of Molecular Structure, 1090, 58-64.

74. Rohani, L., Makita, H., Levitz, A., Henary, M., \& Hastings, G. (2019). Calculated vibrational properties of semiquinones in the A1 binding site in photosystem I. Biochimica et Biophysica Acta (BBA)-Bioenergetics, 1860(9), 699-707.

75. Macaluso, V., Hashem, S., Nottoli, M., Lipparini, F., Cupellini, L., \& Mennucci, B. (2021). Ultrafast transient infrared spectroscopy of photoreceptors with polarizable QM/MM dynamics. The Journal of Physical Chemistry B, 125(36), 10282-10292.

76. Petrone, A., Lingerfelt, D. B., Williams-Young, D. B., \& Li, X. (2016). Ab initio transient vibrational spectral analysis. The Journal of Physical Chemistry Letters, 7(22), 4501-4508.

77. Mezzetti, A., \& Leibl, W. (2017). Time-resolved infrared spectroscopy in the study of photosynthetic systems. Photosynthesis Research, 131(2), 121-144.
78. Gall, A., Berera, R., Alexandre, M. T. A., Pascal, A. A., Bordes, L., Mendes-Pinto, M. M., Andrianambinintsoa, S., Stoitchkova, K. V., Marin, A., Valkunas, L., Horton, P., Kennis, J. T. M., van Grondelle, R., Ruban, A., \& Robert, B. (2011). Molecular adaptation of photoprotection: Triplet states in light-harvesting proteins. Biophysical Journal, 101(4), 934-942.

79. Hastings, G. (2015). Vibrational spectroscopy of photosystem I. Biochimica et Biophysica Acta (BBA)-Bioenergetics, 1847(1), $55-68$.

80. Noguchi, T. (2010). Fourier transform infrared spectroscopy of special pair bacteriochlorophylls in homodimeric reaction centers of heliobacteria and green sulfur bacteria. Photosynthesis Research, 104(2), 321-331.

81. Thibodeau, D. L., Nabedryk, E., Hienerwadel, R., Lenz, F., Mäntele, W., \& Breton, J. (1990). Time-resolved FTIR spectroscopy of quinones in $R b$. sphaeroides reaction centers. Biochimica et Biophysica Acta (BBA)-Bioenergetics, 1020(3), 253-259.

82. Burie, J. R., Leibl, W., Nabedryk, E., \& Breton, J. (1993). Stepscan FT-IR spectroscopy of electron transfer in the photosynthetic bacterial reaction center. Applied Spectroscopy, 47(9), 1401-1404.

83. Hienerwadel, R., Thibodeau, D., Lenz, F., Nabedryk, E., Breton, J., Kreutz, W., \& Maentele, W. (1992). Time-resolved infrared spectroscopy of electron transfer in bacterial photosynthetic reaction centers: Dynamics of binding and interaction upon QA and QB reduction. Biochemistry, 31(25), 5799-5808.

84. Hamm, P., Zurek, M., Mäntele, W., Meyer, M., Scheer, H., \& Zinth, W. (1995). Femtosecond infrared spectroscopy of reaction centers from Rhodobacter sphaeroides between 1000 and 1800 cm-1. Proceedings of the National Academy of Sciences, 92(6), 1826.

85. Wraight, C. A., \& Gunner, M. R. (2009). The acceptor quinones of purple photosynthetic bacteria-structure and spectroscopy. In C. N. Hunter, F. Daldal, M. C. Thurnauer, \& J. T. Beatty (Eds.), The purple phototrophic bacteria (pp. 379-405). Springer Netherlands.

86. Hienerwadel, R., Grzybek, S., Fogel, C., Kreutz, W., Okamura, M. Y., Paddock, M. L., Breton, J., Nabedryk, E., \& Maentele, W. (1995). Protonation of Glu L212 following QB- formation in the photosynthetic reaction center of Rhodobacter sphaeroides: Evidence from time-resolved infrared spectroscopy. Biochemistry, 34(9), 2832-2843.

87. Onidas, D., Stachnik, J. M., Brucker, S., Krätzig, S., \& Gerwert, K. (2010). Histidine is involved in coupling proton uptake to electron transfer in photosynthetic proteins. European Journal of Cell Biology, 89(12), 983-989.

88. Malferrari, M., Mezzetti, A., Francia, F., \& Venturoli, G. (2013). Effects of dehydration on light-induced conformational changes in bacterial photosynthetic reaction centers probed by optical and differential FTIR spectroscopy. Biochimica et Biophysica Acta (BBA)-Bioenergetics, 1827(3), 328-339.

89. Pawlowicz, N. P., van Grondelle, R., van Stokkum, I. H. M., Breton, J., Jones, M. R., \& Groot, M. L. (2008). Identification of the first steps in charge separation in bacterial photosynthetic reaction centers of Rhodobacter sphaeroides by ultrafast midinfrared spectroscopy: Electron transfer and protein dynamics. Biophysical Journal, 95(3), 1268-1284.

90. Pawlowicz, N. P., van Stokkum, I. H. M., Breton, J., van Grondelle, R., \& Jones, M. R. (2009). Identification of the intermediate charge-separated state $\mathrm{P}^{+} \beta_{\mathrm{L}}^{-}$in a leucine M214 to histidine mutant of the Rhodobacter sphaeroides reaction center using femtosecond midinfrared spectroscopy. Biophysical Journal, 96(12), 4956-4965.

91. Pawlowicz, N. P., van Stokkum, I. H. M., Breton, J., van Grondelle, R., \& Jones, M. R. (2010). An investigation of slow charge separation in a Tyrosine M210 to Tryptophan mutant of the 
Rhodobacter sphaeroides reaction center by femtosecond midinfrared spectroscopy. Physical Chemistry Chemical Physics, 12(11), 2693-2705.

92. Stahl, A. D., Crouch, L. I., Jones, M. R., van Stokkum, I., van Grondelle, R., \& Groot, M. L. (2012). Role of PufX in photochemical charge separation in the RC-LH1 complex from Rhodobacter sphaeroides: An ultrafast mid-IR pump-probe investigation. The Journal of Physical Chemistry B, 116(1), 434-444.

93. la Gatta, S., Milano, F., Farinola, G. M., Agostiano, A., Di Donato, M., Lapini, A., Foggi, P., Trotta, M., \& Ragni, R. (2019). A highly efficient heptamethine cyanine antenna for photosynthetic Reaction Center: From chemical design to ultrafast energy transfer investigation of the hybrid system. Biochimica et Biophysica Acta (BBA) Bioenergetics, 1860(4), 350-359.

94. Remy, A., \& Gerwert, K. (2003). Coupling of light-induced electron transfer to proton uptake in photosynthesis. Nature Structural \& Molecular Biology, 10(8), 637-644.

95. Hermes, S., Stachnik, J. M., Onidas, D., Remy, A., Hofmann, E., \& Gerwert, K. (2006). Proton uptake in the reaction center mutant L210DN from Rhodobacter sphaeroides via protonated water molecules. Biochemistry, 45(46), 13741-13749.

96. Breton, J. (2007). Steady-state FTIR spectra of the photoreduction of QA and QB in Rhodobacter sphaeroides reaction centers provide evidence against the presence of a proposed transient electron acceptor $\mathrm{X}$ between the two quinones. Biochemistry, 46(15), 4459-4465.

97. Mezzetti, A., \& Leibl, W. (2005). Investigation of ubiquinol formation in isolated photosynthetic reaction centers by rapid-scan Fourier transform IR spectroscopy. European Biophysics Journal, 34(7), 921-936.

98. Mezzetti, A., \& Leibl, W. (2008). Proton and electron transfer in wild-type and mutant reaction centers from Rhodobacter sphaeroides followed by rapid-scan FTIR spectroscopy. Vibrational Spectroscopy, 48(1), 126-134.

99. Nedelkovski, V., Schwaighofer, A., Wraight, C. A., Nowak, C., \& Naumann, R. L. C. (2013). Surface-enhanced infrared absorption spectroscopy (SEIRAS) of light-activated photosynthetic reaction centers from Rhodobacter sphaeroides reconstituted in a biomimetic membrane system. The Journal of Physical Chemistry C, 117(32), 16357-16363.

100. Groot, M. L., Pawlowicz, N. P., van Wilderen, L. J. G. W., Breton, J., van Stokkum, I. H. M., \& van Grondelle, R. (2005). Initial electron donor and acceptor in isolated Photosystem II reaction centers identified with femtosecond mid-IR spectroscopy. Proceedings of the National Academy of Sciences of the United States of America, 102(37), 13087.

101. Pawlowicz, N. P., Groot, M. L., van Stokkum, I. H. M., Breton, J., \& van Grondelle, R. (2007). Charge separation and energy transfer in the photosystem II core complex studied by femtosecond midinfrared spectroscopy. Biophysical Journal, 93(8), 2732-2742.

102. Kaucikas, M., Maghlaoui, K., Barber, J., Renger, T., \& van Thor, J. J. (2016). Ultrafast infrared observation of exciton equilibration from oriented single crystals of photosystem II. Nature Communications, 7(1), 13977.

103. Noguchi, T., \& Sugiura, M. (2001). Flash-induced fourier transform infrared detection of the structural changes during the $\mathrm{S}$-state cycle of the oxygen-evolving complex in photosystem II. Biochemistry, 40(6), 1497-1502.

104. Hillier, W., \& Babcock, G. T. (2001). S-state dependent fourier transform infrared difference spectra for the photosystem II oxygen evolving complex. Biochemistry, 40(6), 1503-1509.

105. Kimura, Y., Ishii, A., Yamanari, T., \& Ono, T.-A. (2005). Watersensitive low-frequency vibrations of reaction intermediates during S-state cycling in photosynthetic water oxidation. Biochemistry, 44(21), 7613-7622.
106. Shimizu, T., Sugiura, M., \& Noguchi, T. (2018). Mechanism of proton-coupled electron transfer in the S0-to-S1 transition of photosynthetic water oxidation as revealed by time-resolved infrared spectroscopy. The Journal of Physical Chemistry B, 122(41), 9460-9470.

107. Sakamoto, H., Shimizu, T., Nagao, R., \& Noguchi, T. (2017). Monitoring the reaction process during the $\mathrm{S} 2 \rightarrow \mathrm{S} 3$ transition in photosynthetic water oxidation using time-resolved infrared spectroscopy. Journal of the American Chemical Society, 139(5), 2022-2029.

108. Yata, H., \& Noguchi, T. (2018). Mechanism of methanol inhibition of photosynthetic water oxidation as studied by Fourier transform infrared difference and time-resolved infrared spectroscopies. Biochemistry, 57(32), 4803-4815.

109. Shimada, Y., Kitajima-Ihara, T., Nagao, R., \& Noguchi, T. (2020). Role of the O4 channel in photosynthetic water oxidation as revealed by fourier transform infrared difference and timeresolved infrared analysis of the D1-S169A Mutant. The Journal of Physical Chemistry B, 124(8), 1470-1480.

110. Takemoto, H., Sugiura, M., \& Noguchi, T. (2019). Proton release process during the S2-to-S3 transition of photosynthetic water oxidation as revealed by the $\mathrm{pH}$ dependence of kinetics monitored by time-resolved infrared spectroscopy. Biochemistry, 58(42), 4276-4283.

111. Okamoto, Y., Shimada, Y., Nagao, R., \& Noguchi, T. (2021). Proton and water transfer pathways in the $\mathrm{S} 2 \rightarrow \mathrm{S} 3$ transition of the water-oxidizing complex in photosystem II: Time-resolved infrared analysis of the effects of D1-N298A mutation and NO3substitution. The Journal of Physical Chemistry B, 125(25), 6864-6873.

112. Mäusle, S. M., Abzaliyeva, A., Greife, P., Simon, P. S., Perez, R., Zilliges, Y., \& Dau, H. (2020). Activation energies for two steps in the $\mathrm{S} 2 \rightarrow \mathrm{S} 3$ transition of photosynthetic water oxidation from time-resolved single-frequency infrared spectroscopy. The Journal of Chemical Physics, 153(21), 215101.

113. Suga, M., Akita, F., Hirata, K., Ueno, G., Murakami, H., Nakajima, Y., Shimizu, T., Yamashita, K., Yamamoto, M., Ago, H., \& Shen, J.-R. (2015). Native structure of photosystem II at 1.95 $\AA$ resolution viewed by femtosecond X-ray pulses. Nature, 517(7532), 99-103.

114. Nozawa, Y., \& Noguchi, T. (2018). pH-dependent regulation of the relaxation rate of the radical anion of the secondary quinone electron acceptor QB in photosystem II as revealed by fourier transform infrared spectroscopy. Biochemistry, 57(19), $2828-2836$.

115. Sipka, G., Magyar, M., Mezzetti, A., Akhtar, P., Zhu, Q., Xiao, Y., Han, G., Santabarbara, S., Shen, J.-R., Lambrev, P. H., \& Garab, G. (2021). Light-adapted charge-separated state of photosystem II: Structural and functional dynamics of the closed reaction center. The Plant Cell, 33(4), 1286-1302.

116. Sato, A., Nakano, Y., Nakamura, S., \& Noguchi, T. (2021). Rapid-scan time-resolved ATR-FTIR study on the photoassembly of the water-oxidizing $\mathrm{Mn} 4 \mathrm{CaO} 5$ cluster in photosystem II. The Journal of Physical Chemistry B, 125(16), 4031-4045.

117. Wang, W., Zheng, Y., Lin, J., She, Y., \& Fu, K. J. (1993). Timeresolved IR study of gas-phase reactions of benzene with Group VIB metal pentacarbonyls and tetracarbonyls. The Journal of Physical Chemistry, 97(46), 11921-11928.

118. Wang, W., Chen, F., Lin, J., \& She, Y. (1995). Time-resolved IR studies of gas-phase photochemistry of $\mathrm{Co}(\mathrm{CO}) 3 \mathrm{NO}$. Journal of the Chemical Society, Faraday Transactions, 91(5), 847-853.

119. Yeom, Y.-H., \& Frei, H. (2002). Mechanistic study of $\mathrm{CH} 3 \mathrm{OH}$ $+\mathrm{O} 2$ photoredox reaction in a FeAlPO4 sieve by time-resolved FT-IR spectroscopy. The Journal of Physical Chemistry A, 106(14), 3350-3355. 
120. Yamakata, A., Ishibashi, T.-A., \& Onishi, H. (2003). Kinetics of the photocatalytic water-splitting reaction on $\mathrm{TiO} 2$ and $\mathrm{Pt} /$ $\mathrm{TiO} 2$ studied by time-resolved infrared absorption spectroscopy. Journal of Molecular Catalysis A: Chemical, 199(1), 85-94.

121. Chen, T., Feng, Z., Wu, G., Shi, J., Ma, G., Ying, P., \& Li, C. (2007). Mechanistic Studies of Photocatalytic Reaction of Methanol for Hydrogen Production on $\mathrm{Pt} / \mathrm{TiO} 2$ by in situ Fourier transform IR and time-resolved IR spectroscopy. The Journal of Physical Chemistry C, 111(22), 8005-8014.

122. Schnee, J., Daturi, M., \& El-Roz, M. (2020). Ultrafast timeresolved quantum cascade laser diagnostic for revealing the role of surface formate species in the photocatalytic oxidation of methanol. Catalysis Science \& Technology, 10(16), 5618-5627.

123. El-Roz, M., Bazin, P., \& Thibault-Starzyk, F. (2013). An operando-IR study of photocatalytic reaction of methanol on new *BEA supported TiO2 catalyst. Catalysis Today, 205, 111-119.

124. Ryczkowski, J. (2001). IR spectroscopy in catalysis. Catalysis Today, 68(4), 263-381.

125. El-Roz, M., Kus, M., Cool, P., \& Thibault-Starzyk, F. (2012). New operando IR technique to study the photocatalytic activity and selectivity of $\mathrm{TiO} 2$ nanotubes in air purification: Influence of temperature, UV intensity, and VOC CONCENTRATION. The Journal of Physical Chemistry C, 116(24), 13252-13263.

126. Blatter, F., Sun, H., \& Frei, H. (1996). Highly Selective Formation of tert-Butyl Hydroperoxide from the Reaction of Isobutane and $\mathrm{O} 2$ in a Zeolite under Visible Light. Chemistry-A European Journal, 2(4), 385-389.

127. Yamakata, A., Ishibashi, T.-A., \& Onishi, H. (2001). Timeresolved infrared absorption spectroscopy of photogenerated electrons in platinized TiO2 particles. Chemical Physics Letters, 333(3), 271-277.

128. Yamakata, A., Ishibashi, T.-A., \& Onishi, H. (2001). Water- and oxygen-induced decay kinetics of photogenerated electrons in $\mathrm{TiO} 2$ and $\mathrm{Pt} / \mathrm{TiO} 2$ : A time-resolved infrared absorption study. The Journal of Physical Chemistry B, 105(30), 7258-7262.

129. Yamakata, A., Ishibashi, T.-A., \& Onishi, H. (2002). Electronand hole-capture reactions on $\mathrm{Pt} / \mathrm{TiO} 2$ photocatalyst exposed to methanol vapor studied with time-resolved infrared absorption spectroscopy. The Journal of Physical Chemistry B, 106(35), 9122-9125.

130. Al-Mazroai, L. S., Bowker, M., Davies, P., Dickinson, A., Greaves, J., James, D., \& Millard, L. (2007). The photocatalytic reforming of methanol. Catalysis Today, 122(1), 46-50.

131. Vasenkov, S., \& Frei, H. (1998). Time-resolved FT-infrared spectroscopy of visible light-induced alkene oxidation by $\mathrm{O} 2$ in a zeolite. The Journal of Physical Chemistry B, 102(42), 8177-8182.

132. Ulagappan, N., \& Frei, H. (2000). Redox chemistry of gaseous reactants inside photoexcited FeAlPO4 molecular sieve. The Journal of Physical Chemistry A, 104(3), 490-496.

133. Ulagappan, N., \& Frei, H. (2000). Mechanistic study of CO2 photoreduction in Ti silicalite molecular sieve by FT-IR spectroscopy. The Journal of Physical Chemistry A, 104(33), 7834-7839.

134. Sun, H., Blatter, F., \& Frei, H. (1996). Cyclohexanone from cyclohexane and $\mathrm{O} 2$ in a zeolite under visible light with complete selectivity. Journal of the American Chemical Society, 118(29), 6873-6879.

135. Macnaughtan, M. L., Soo, H. S., \& Frei, H. (2014). Binuclear ZrOCo metal-to-metal charge-transfer unit in mesoporous silica for light-driven $\mathrm{CO} 2$ reduction to $\mathrm{CO}$ and formate. The Journal of Physical Chemistry C, 118(15), 7874-7885.

136. Lin, W., Han, H., \& Frei, H. (2004). CO2 Splitting by $\mathrm{H} 2 \mathrm{O}$ to $\mathrm{CO}$ and $\mathrm{O} 2$ under UV Light in TiMCM-41 Silicate Sieve. The Journal of Physical Chemistry B, 108(47), 18269-18273.

137. Blatter, F., \& Frei, H. (1994). Selective photooxidation of small alkenes by $\mathrm{O} 2$ with red light in zeolite Y. Journal of the American Chemical Society, 116(5), 1812-1820.
138. Yeom, Y. H., Ulagappan, N., \& Frei, H. (2002). Chemical reactivity of formaldehyde in a FeAlPO4 sieve. The Journal of Physical Chemistry A, 106(14), 3345-3349.

139. Feringa, B. L., \& Browne, W. R. (2011). Molecular Switches (2nd ed.). Wiley-VCH.

140. Laptenok, S. P., Lukacs, A., Gil, A., Brust, R., Sazanovich, I. V., Greetham, G. M., Tonge, P. J., \& Meech, S. R. (2015). Complete proton transfer cycle in GFP and Its T203V and S205V mutants. Angewandte Chemie International Edition, 54(32), 9303-9307.

141. Di Donato, M., van Wilderen, L. J. G. W., Van Stokkum, I. H. M., Stuart, T. C., Kennis, J. T. M., Hellingwerf, K. J., van Grondelle, R., \& Groot, M. L. (2011). Proton transfer events in GFP. Physical Chemistry Chemical Physics, 13(36), 16295-16305.

142. Cheng, C.-W., Huang, G.-J., Hsu, H.-Y., Prabhakar, C., Lee, Y.-P., Diau, E.W.-G., \& Yang, J.-S. (2013). Effects of hydrogen bonding on internal conversion of GFP-like chromophores. II. The meta-amino systems. The Journal of Physical Chemistry B, 117(9), 2705-2716.

143. Hsieh, C.-C., Chou, P.-T., Shih, C.-W., Chuang, W.-T., Chung, M.-W., Lee, J., \& Joo, T. (2011). Comprehensive studies on an overall proton transfer cycle of the ortho-green fluorescent protein chromophore. Journal of the American Chemical Society, 133(9), 2932-2943.

144. van Thor, J. J., Georgiev, G. Y., Towrie, M., \& Sage, J. T. (2005). Ultrafast and low barrier motions in the photoreactions of the green fluorescent protein. Journal of Biological Chemistry, 280(39), 33652-33659.

145. van Wilderen, L. J. G. W., van der Horst, M. A., van Stokkum, I. H. M., Hellingwerf, K. J., van Grondelle, R., \& Groot, M. L. (2006). Ultrafast infrared spectroscopy reveals a key step for successful entry into the photocycle for photoactive yellow protein. Proceedings of the National Academy of Sciences, 103(41), $15050-15055$.

146. Rupenyan, A. B., Vreede, J., van Stokkum, I. H. M., Hospes, M., Kennis, J. T. M., Hellingwerf, K. J., \& Groot, M. L. (2011). Proline 68 enhances photoisomerization yield in photoactive yellow protein. The Journal of Physical Chemistry B, 115(20), 6668-6677.

147. Briand, J., Léonard, J., \& Haacke, S. (2010). Ultrafast photoinduced reaction dynamics in bacteriorhodopsin and its Trp mutants. Journal of Optics, 12(8), 084004.

148. Diller, R., Jakober, R., Schumann, C., Peters, F., Klare, J. P., \& Engelhard, M. (2006). The trans-cis isomerization reaction dynamics in sensory rhodopsin II by femtosecond time-resolved midinfrared spectroscopy: Chromophore and protein dynamics. Biopolymers, 82(4), 358-362.

149. Verhoefen, M.-K., Neumann, K., Weber, I., Glaubitz, C., \& Wachtveitl, J. (2009). Primary reaction dynamics of proteorhodopsin mutant D97N observed by femtosecond infrared and visible spectroscopy. Photochemistry and Photobiology, 85(2), 540-546.

150. Stensitzki, T., Yang, Y., Muders, V., Schlesinger, R., Heberle, J., $\&$ Heyne, K. (2016). Femtosecond infrared spectroscopy of channelrhodopsin-1 chromophore isomerization. Structural Dynamics, 3(4), 043208.

151. Peters, F., Herbst, J., Tittor, J., Oesterhelt, D., \& Diller, R. (2006). Primary reaction dynamics of halorhodopsin, observed by subpicosecond IR-Vibrational spectroscopy. Chemical Physics, 323(1), 109-116.

152. Herbst, J., Heyne, K., \& Diller, R. (2002). Femtosecond infrared spectroscopy of bacteriorhodopsin chromophore isomerization. Science, 297(5582), 822-825.

153. Hamm, P., Ohline, S. M., \& Zinth, W. (1997). Vibrational cooling after ultrafast photoisomerization of azobenzene measured by femtosecond infrared spectroscopy. The Journal of Chemical Physics, 106(2), 519-529. 
154. Wiedbrauk, S., \& Dube, H. (2015). Hemithioindigo-an emerging photoswitch. Tetrahedron Letters, 56(29), 4266-4274.

155. Stallhofer, K., Nuber, M., Schüppel, F., Thumser, S., Iglev, H., de Vivie-Riedle, R., Zinth, W., \& Dube, H. (2021). Electronic and geometric characterization of TICT formation in hemithioindigo photoswitches by picosecond infrared spectroscopy. The Journal of Physical Chemistry A, 125(20), 4390-4400.

156. Amirjalayer, S., Cnossen, A., Browne, W. R., Feringa, B. L., Buma, W. J., \& Woutersen, S. (2016). Direct observation of a dark state in the photocycle of a light-driven molecular motor. The Journal of Physical Chemistry A, 120(43), 8606-8612.

157. Helmy, S., Leibfarth, F. A., Oh, S., Poelma, J. E., Hawker, C. J., \& Read de Alaniz, J. (2014). Photoswitching using visible light: A new class of organic photochromic molecules. Journal of the American Chemical Society, 136(23), 8169-8172.

158. Helmy, S., Oh, S., Leibfarth, F. A., Hawker, C. J., \& Read de Alaniz, J. (2014). Design and synthesis of donor-acceptor stenhouse adducts: A visible light photoswitch derived from furfural. The Journal of Organic Chemistry, 79(23), 11316-11329.

159. Helmy, S., \& Read de Alaniz, J. (2015). Chapter three-photochromic and thermochromic heterocycles. In E. F. V. Scriven \& C. A. Ramsden (Eds.), Advances in heterocyclic chemistry (Vol. 117, pp. 131-177). Academic Press.

160. Lerch, M. M., Szymański, W., \& Feringa, B. L. (2018). The (photo)chemistry of Stenhouse photoswitches: Guiding principles and system design. Chemical Society Reviews, 47(6), 1910-1937.

161. Lerch, M. M., Wezenberg, S. J., Szymanski, W., \& Feringa, B. L. (2016). Unraveling the photoswitching mechanism in donoracceptor stenhouse adducts. Journal of the American Chemical Society, 138(20), 6344-6347.

162. Di Donato, M., Lerch, M. M., Lapini, A., Laurent, A. D., Iagatti, A., Bussotti, L., Ihrig, S. P., Medved', M., Jacquemin, D., Szymański, W., Buma, W. J., Foggi, P., \& Feringa, B. L. (2017). Shedding light on the photoisomerization pathway of donoracceptor stenhouse adducts. Journal of the American Chemical Society., 139(44), 15596-15599.

163. Zulfikri, H., Koenis, M. A. J., Lerch, M. M., Di Donato, M., Szymański, W., Filippi, C., Feringa, B. L., \& Buma, W. J. (2019). Taming the complexity of donor-acceptor stenhouse adducts: Infrared motion pictures of the complete switching pathway. Journal of the American Chemical Society, 141(18), 7376-7384.

164. Patricia, T. T., Sandra, M. V., Manuela, L., Andrea, L., Paolo, F., Andrea, D., \& Roberto, R. (2012). Transient infrared spectroscopy: A new approach to investigate valence tautomerism. Physical Chemistry Chemical Physics, 14(2), 1038-1047.

165. Best, J., Sazanovich, I. V., Adams, H., Bennett, R. D., Davies, E. S., Meijer, A. J. H. M., Towrie, M., Tikhomirov, S. A., Bouganov, O. V., Ward, M. D., \& Weinstein, J. A. (2010). Structure and ultrafast dynamics of the charge-transfer excited state and redox activity of the ground state of mono- and binuclear platinum(II) diimine catecholate and bis-catecholate complexes: A transient absorption, TRIR, DFT, and electrochemical study. Inorganic Chemistry, 49(21), 10041-10056.

166. Li, G., Parimal, K., Vyas, S., Hadad, C. M., Flood, A. H., \& Glusac, K. D. (2009). Pinpointing the extent of electronic delocalization in the $\operatorname{Re}(\mathrm{I})$-to-tetrazine charge-separated excited state using time-resolved infrared spectroscopy. Journal of the American Chemical Society, 131(33), 11656-11657.

167. Maçôas, E. M. S., Kananavicius, R., Myllyperkiö, P., Pettersson, M., \& Kunttu, H. (2007). Ultrafast electronic and vibrational energy relaxation of $\mathrm{Fe}$ (acetylacetonate) 3 in solution. The Journal of Physical Chemistry A, 111(11), 2054-2061.

168. Yue, Y., Grusenmeyer, T., Ma, Z., Zhang, P., Schmehl, R. H., Beratan, D. N., \& Rubtsov, I. V. (2014). Full-electron ligand-to-ligand charge transfer in a compact $\operatorname{Re}(\mathrm{I})$ complex. The Journal of Physical Chemistry A, 118(45), 10407-10415.

169. Asbury, J. B., Hao, E., Wang, Y., \& Lian, T. (2000). Bridge length-dependent ultrafast electron transfer from re polypyridyl complexes to nanocrystalline $\mathrm{TiO} 2$ thin films studied by femtosecond infrared spectroscopy. The Journal of Physical Chemistry $B, 104(50), 11957-11964$.

170. Furube, A., Katoh, R., Hara, K., Sato, T., Murata, S., Arakawa, H., \& Tachiya, M. (2005). Lithium ion effect on electron injection from a photoexcited coumarin derivative into a $\mathrm{TiO} 2$ nanocrystalline film investigated by visible-to-IR ultrafast spectroscopy. The Journal of Physical Chemistry B, 109(34), 16406-16414.

171. Paoprasert, P., Laaser, J. E., Xiong, W., Franking, R. A., Hamers, R. J., Zanni, M. T., Schmidt, J. R., \& Gopalan, P. (2010). Bridgedependent interfacial electron transfer from rhenium-bipyridine complexes to $\mathrm{TiO} 2$ nanocrystalline thin films. The Journal of Physical Chemistry C, 114(21), 9898-9907.

172. Juozapavicius, M., Kaucikas, M., van Thor, J. J., \& O’Regan, B. C. (2013). Observation of multiexponential pico- to subnanosecond electron injection in optimized dye-sensitized solar cells with visible-pump mid-infrared-probe transient absorption spectroscopy. The Journal of Physical Chemistry C, 117(1), 116-123.

173. Horvath, R., Fraser, M. G., Clark, C. A., Sun, X.-Z., George, M. W., \& Gordon, K. C. (2015). Nature of excited states of ruthenium-based solar cell dyes in solution: A comprehensive spectroscopic study. Inorganic Chemistry, 54(24), 11697-11708.

174. Xiong, W., Laaser, J. E., Paoprasert, P., Franking, R. A., Hamers, R. J., Gopalan, P., \& Zanni, M. T. (2009). Transient 2D IR spectroscopy of charge injection in dye-sensitized nanocrystalline thin films. Journal of the American Chemical Society, 131(50), 18040-18041.

175. Pankove, J. I. (1975). Optical processes in semiconductors. Courier Corporation.

176. Diesinger, H., Chan, E. A., Yin, J., \& Soci, C. (2013). 11-Ultrafast charge carrier dynamics in organic (opto)electronic materials. In O. Ostroverkhova (Ed.), Handbook of organic materials for optical and (Opto)electronic devices (pp. 318-355). Woodhead Publishing.

177. He, X., Zhu, G., Yang, J., Chang, H., Meng, Q., Zhao, H., Zhou, X., Yue, S., Wang, Z., Shi, J., Gu, L., Yan, D., \& Weng, Y. (2015). Photogenerated intrinsic free carriers in small-molecule organic semiconductors visualized by ultrafast spectroscopy. Scientific Reports, 5(1), 17076.

178. Yeh, T.-T., Shirai, H., Tu, C.-M., Fuji, T., Kobayashi, T., \& Luo, C.-W. (2017). Ultrafast carrier dynamics in Ge by ultra-broadband mid-infrared probe spectroscopy. Scientific Reports, 7(1), 40492.

179. Li, X., Zhou, D., Hao, H., Chen, H., Weng, Y., \& Bian, H. (2020). Vibrational relaxation dynamics of a semiconductor copper(I) thiocyanate (CuSCN) film as a hole-transporting layer. The Journal of Physical Chemistry Letters, 11(2), 548-555.

180. Moses, D., Dogariu, A., \& Heeger, A. J. (2000). Ultrafast detection of charged photocarriers in conjugated polymers. Physical Review B, 61(14), 9373-9379.

181. Narra, S., Chung, C.-C., Diau, E.W.-G., \& Shigeto, S. (2016). Simultaneous observation of an intraband transition and distinct transient species in the infrared region for perovskite solar cells. The Journal of Physical Chemistry Letters, 7(13), 2450-2455.

182. Guo, P., Gong, J., Sadasivam, S., Xia, Y., Song, T.-B., Diroll, B. T., Stoumpos, C. C., Ketterson, J. B., Kanatzidis, M. G., Chan, M. K. Y., Darancet, P., Xu, T., \& Schaller, R. D. (2018). Slow thermal equilibration in methylammonium lead iodide revealed by transient mid-infrared spectroscopy. Nature Communications, 9(1), 2792. 
183. Taylor, V. C. A., Tiwari, D., Duchi, M., Donaldson, P. M., Clark, I. P., Fermin, D. J., \& Oliver, T. A. A. (2018). Investigating the role of the organic cation in formamidinium lead iodide perovskite using ultrafast spectroscopy. The Journal of Physical Chemistry Letters, 9(4), 895-901.

184. Guo, Z., Wan, Y., Yang, M., Snaider, J., Zhu, K., \& Huang, L. (2017). Long-range hot-carrier transport in hybrid perovskites visualized by ultrafast microscopy. Science, 356(6333), 59-62.

185. Munson, K. T., Kennehan, E. R., Doucette, G. S., \& Asbury, J. B. (2018). Dynamic disorder dominates delocalization, transport, and recombination in halide perovskites. Chem, 4(12), 2826-2843.

186. Munson, K. T., Doucette, G. S., Kennehan, E. R., Swartzfager, J. R., \& Asbury, J. B. (2019). Vibrational probe of the structural origins of slow recombination in halide perovskites. The Journal of Physical Chemistry C, 123(12), 7061-7073.

187. Guo, P., Mannodi-Kanakkithodi, A., Gong, J., Xia, Y., Stoumpos, C. C., Cao, D. H., Diroll, B. T., Ketterson, J. B., Wiederrecht, G. P., Xu, T., Chan, M. K. Y., Kanatzidis, M. G., \& Schaller, R.
D. (2019). Infrared-pump electronic-probe of methylammonium lead iodide reveals electronically decoupled organic and inorganic sublattices. Nature Communications, 10(1), 482.

188. Norahan, M. J., Horvath, R., Woitzik, N., Jouy, P., Eigenmann, F., Gerwert, K., \& Kötting, C. (2021). Microsecond-resolved infrared spectroscopy on nonrepetitive protein reactions by applying caged compounds and quantum cascade laser frequency combs. Analytical Chemistry, 93(17), 6779-6783.

189. Sanchez, M. L. K., Sommer, C., Reijerse, E., Birrell, J. A., Lubitz, W., \& Dyer, R. B. (2019). Investigating the kinetic competency of CrHydA $1[\mathrm{FeFe}]$ hydrogenase intermediate states via time-resolved infrared spectroscopy. Journal of the American Chemical Society, 141(40), 16064-16070.

190. Hashimoto, K., Badarla, V. R., \& Ideguchi, T. (2021). High-speed Fourier-transform infrared spectroscopy with phase-controlled Delay Line. Laser \& Photonics Reviews, 15(1), 2000374.

191. Kawai, A., Hashimoto, K., Dougakiuchi, T., Badarla, V. R., Imamura, T., Edamura, T., \& Ideguchi, T. (2020). Time-stretch infrared spectroscopy. Communications Physics, 3(1), 152. 